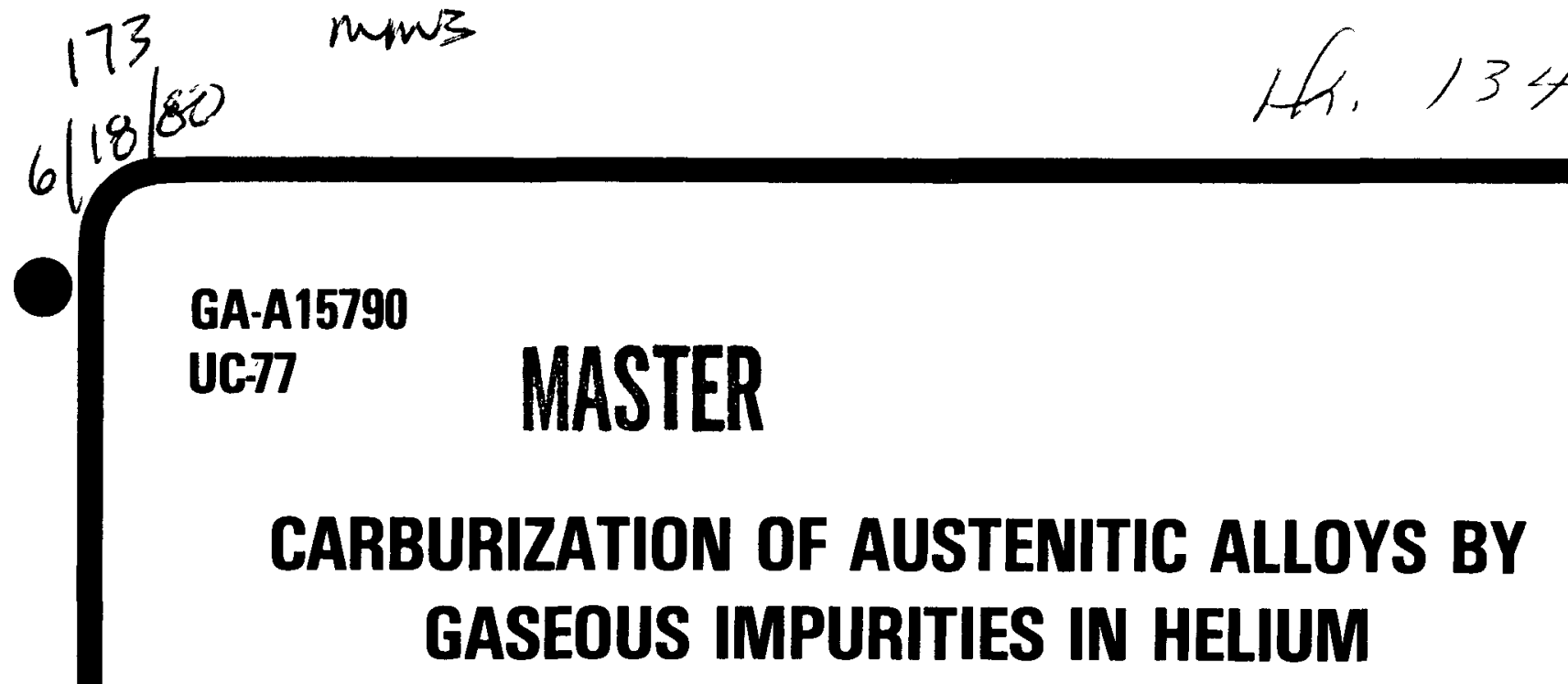

by
G. Y. LAl and W. R. JOHNSON

DATE PUBLISHED: MARCH 1980 


\section{DISCLAIMER}

This report was prepared as an account of work sponsored by an agency of the United States Government. Neither the United States Government nor any agency Thereof, nor any of their employees, makes any warranty, express or implied, or assumes any legal liability or responsibility for the accuracy, completeness, or usefulness of any information, apparatus, product, or process disclosed, or represents that its use would not infringe privately owned rights. Reference herein to any specific commercial product, process, or service by trade name, trademark, manufacturer, or otherwise does not necessarily constitute or imply its endorsement, recommendation, or favoring by the United States Government or any agency thereof. The views and opinions of authors expressed herein do not necessarily state or reflect those of the United States Government or any agency thereof. 


\section{DISCLAIMER}

Portions of this document may be illegible in electronic image products. Images are produced from the best available original document. 
GA-A15790

UC77

\title{
CARBURIZATION OF AUSTENITIC ALLOYS BY GASEOUS IMPURITIES IN HELIUM
}

\author{
by \\ G. Y. LAI and W. R. JOHNSON
}

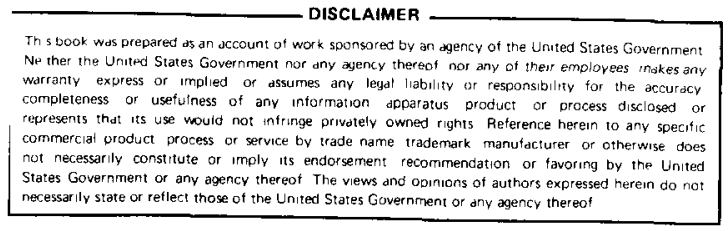

This is a preprint of a paper to be submitted for publication in Metallurgical Transactions.

Work supported by

Department of Energy

Under Contract DE-AT03-76ET35300

GENERAL ATOMIC PROJECT 6400

DATE PUBLISHED: MARCH 1980

\section{GENERAL ATOMIC COMPANY}




\section{ABSTRACT}

The carburization behavior of Alloy 800H, Inconel Alloy 617 and Hastelloy Alloy $\mathrm{X}$ in helium containing various amounts of $\mathrm{H}_{2}, \mathrm{CO}, \mathrm{CH}_{4}, \mathrm{H}_{2} \mathrm{O}$ and $\mathrm{CO}_{2}$ was studied. Corrosion tests were conducted in a temperature range from $649^{\circ}$ to $1000^{\circ} \mathrm{C}\left(1200^{\circ}\right.$ to $\left.1832^{\circ} \mathrm{F}\right)$ for exposure time up to $10,000 \mathrm{~h}$. Four different helium environments, identified as A, B, C and D, were investigated. Concentrations of gaseous impurities were $1500 \mu \mathrm{atm} \mathrm{H}$,

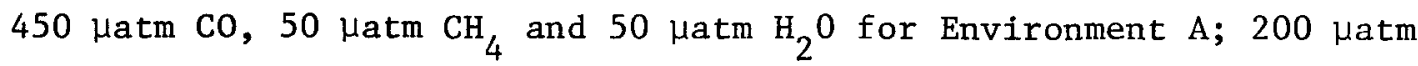

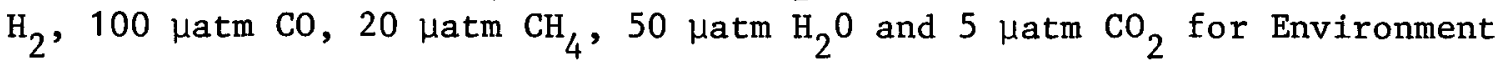

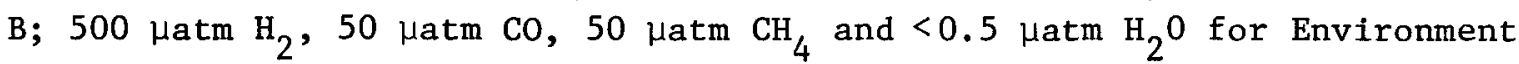
$\mathrm{C}$; and $500 \mu \mathrm{atm} \mathrm{H}_{2}, 50$ atm $\mathrm{CO}, 50 \mu \mathrm{atm} \mathrm{CH}_{4}$ and $1.5 \mu \mathrm{atm} \mathrm{H}_{2} \mathrm{O}$ for Environment D. Environments A and B were characteristic of high-oxygen potential, while $C$ and $D$ were characteristic of low-oxygen potential. The results showed that the carburization kinetics in low-oxygen potential environments (C and D) were significantly higher, approximately an order of magnitude higher at high temperatures, than those in high-oxygen potential environments ( $A$ and $B$ ) for all three alloys. Thermodynamic analyses indicated no significant differences in the thermodynamic carburization potential between low- and high-oxygen potential environments. It is thus believed that the enhanced carburization kinetics observed in the low-oxygen potential environments were related to kinetic effects. A qualitatively mechanistic model was proposed to explain the enhanced kinetics. The present results further suggest that controlling the oxygen potential of the service environment can be an effective means of reducing carburization of alloys. 
ABSTRACT . . . . . . . . . . . . . . . . . . . . $i i i$

1. INTRODUCTION . . . . . . . . . . . . . . . . . 1

2. MATERIALS AND EXPERIMENTS ................... . . . 3

3. EXPERIMENTAL RESULTS . . . . . . . . . . . . . . . . . 9

4. DISCUSSION . . . . . . . . . . . . . . . . . . 20

5. SUMMARY AND CONCLUSIONS . . . . . . . . . . . . . . . 38

6. ACKNOWLEDGMENTS . . . . . . . . . . . . . . . . . 40

7. REFERENCES ....................... . . . 41

\section{FIGURES}

1. Mass of carbon increase as a result of exposure in Environments $\mathrm{C} / \mathrm{D}$ at various temperatures and times for Hastelloy Alloy

$\mathrm{X}$ : (a) Heat C-15567, (b) Heat 5-2831 . . . . . . . . . . 14

2. Rates of carbon increase in Alloy $800 \mathrm{H}$ as a result of exposure to Environments $A, B$ and $D$ as a function of inverse of exposure temperatures (absolute temperature) . . . . . 16

3. Rates of carbon increase in Inconel Alloy 617 as a result of exposure to Environments B, C and D as a function of inverse of exposure temperatures (absolute temperature)...

4. Rates of carbon increase in Hastelloy Alloy $X$ as a result of exposure to Environments A, B, C and D as a function of inverse of exposure temperatures (absolute temperatures) . 18

5. Calculated carbon activities in the test environment for various temperatures based on the reaction: $\mathrm{CO}+\mathrm{H}_{2} \rightleftharpoons \mathrm{C}+\mathrm{H}_{2} 0$. Also plotted are calculated carbon activities in equilibrium with $\mathrm{Cr}_{23} \mathrm{C}_{6}$ in indicated alloys . . . . . . .

6. Calculated carbon activities in the test environment for various temperatures based on the reaction: $2 \mathrm{CO} \rightleftharpoons \mathrm{C}+\mathrm{CO}_{2}$. Also plotted are calculated carbon activities in equilibrium with $\mathrm{Cr}_{23} \mathrm{C}_{6}$ in indicated alloys . . . . . . . . . . 23

7. Calculated carbon activities in the test environment for various temperatures based on the reaction: $\mathrm{CH}_{4} \rightleftharpoons \mathrm{C}+2 \mathrm{H}_{2}$. Carbon activities in austenitic alloys not plotted in the figure are well below the range in the figure . . . . . . 
8. Calculated carbon activities in the test environment for various temperatures based on the reaction: $\mathrm{CH}_{4}+\mathrm{CO} \rightleftharpoons 2 \mathrm{C}+\mathrm{H}_{2} \mathrm{O}+\mathrm{H}_{2}$. Carbon activities in austenitic alloys not plotted in ${ }^{2}$ the figure are well below the range in the figure . . . . . 25

9. Calculated partial pressure of $\mathrm{CO}$ in equilibrium with $\mathrm{Cr}$, $\mathrm{Cr}_{23} \mathrm{C}_{6}$ and $\mathrm{Cr}_{2} \mathrm{O}_{3}$ at indicated $\mathrm{Cr}$ activities in accordance with the reaction $27 \mathrm{Cr}+6 \mathrm{CO} \rightleftharpoons \mathrm{Cr}_{23} \mathrm{C}_{6}+2 \mathrm{Cr}_{2} \mathrm{O}_{3}$ as a function of $1 / T$. Also plotted are partial pressures for Environments A, $\mathrm{B}, \mathrm{C}$ and $\mathrm{D}$. . . . . . . . . . . . . . . . .

10. Equilibrium oxygen partial pressures for various metallic oxides as a function of $1 / \mathrm{T}\left(\mathrm{K}^{-1}\right)$. Also plotted in the figure are calculated oxygen partial pressures for Environments $A$, $B, C$ and $D$ based on the reation: $\mathrm{H}_{2} \mathrm{O} \rightleftharpoons 1 / 2 \mathrm{O}_{2}+\mathrm{H}_{2} \cdot$... .

11. The stability diagram for the $\mathrm{Cr}-0-\mathrm{C}$ system at $900^{\circ} \mathrm{C}\left(1650^{\circ} \mathrm{F}\right)$. Also shown in the figure are calculated oxygen partial pressures in Environments A, B, C and D . . . . . . . . .

12. Optical photomicrographs showing the typical oxide scale formed on the metal surface of Alloy $800 \mathrm{H}$, Inconel Alloy 617 and Hastelloy Alloy $X$ after exposure to environment $A$ at $871^{\circ} \mathrm{C}$ $\left(1600^{\circ} \mathrm{F}\right)$ for $5000 \mathrm{~h}(\mathrm{a}, \mathrm{b}, \mathrm{c})$ and to environment $\mathrm{B}$ at $900^{\circ} \mathrm{C}$ $\left(1650^{\circ} \mathrm{F}\right)$ for $5000 \mathrm{~h}(\mathrm{~d}, \mathrm{e}, \mathrm{f})$. The phases indicated by the arrow were found to be chromium-depleted metal matrix phases. Unetched . . . . . . . . . . . . . . .

13. Optical photomicrographs showing the typical oxide-carbide duplex scale formed on the metal surface of Alloy $800 \mathrm{H}$ (a), Inconel Alloy 617 (b), and Hastelloy Alloy X (c) after exposure to environment $\mathrm{C}$ at $900^{\circ} \mathrm{C}\left(1650^{\circ} \mathrm{F}\right)$ for $3000 \mathrm{~h}$. Unetched . .

14. Schematics of the qualitatively mechanistic model for carburization of metals in high-oxygen potential environments (A and $B$ ) and low-oxygen potential environments

(C and D).........................

\section{TABLES}

1. Exposure conditions for the alloys under investigation . . .

2. Heat number, form, and grain size of alloys tested in each environment.................... . . . . 6

3. Chemical composition of alloys under investigation . . . . . 7

4. Results of bulk carbon analysis on specimens exposed in Environment A . . . . . . . . . . . . . . . . . 


\section{TABLES (continued)}

5. Results of bulk carbon analysis on specimens exposed in Environment B . . . . . . . . . . . . . . . . .

6. Results of bulk carbon analysis on specimens exposed in Environment C . . . . . . . . . . . . . . 12

7. Results of bulk carbon analysis on specimens exposed in Environment D . . . . . . . . . . . . . . . . . . 


\section{INTRODUCTION}

Helium is used as a primary coolant in high-temperature gas-cooled reactors (HTGRs) designed in the U.S., Germany, Japan, etc. Because it is chemically inert, helium cannot react with structural materials under normal or accident conditions. However, the primary coolant helium in an operating gas-cooled reactor can be contaminated by small amounts (parts per million levels) of gaseous impurities. The principal impurities are $\mathrm{H}_{2}, \mathrm{CO}, \mathrm{CH}_{4}$ and $\mathrm{H}_{2} \mathrm{O}$. These impurities may arise from a variety of sources, such as water and steam ingress, reactions of ingressed steam with the core graphite, outgassing of reactor materials, proton diffusion through steam tubes, oil ingress, etc. Corrosion of metals by these gaseous impurities at elevated temperatures can be quite significant. Recent studies (Refs. 1-5) have indicated that the corrosion of austenitic alloys by these gaseous impurities includes both oxidation and carburization. Carburization is believed to be a more important mode of corrosion in affecting the material performance. Current understanding of these gas-metal interactions, particularly their mechanistic and kinetic aspects, however, is still limited. Extensive work is required to fully understand the corrosion behavior and how these corrosive environments affect the long-term mechanical properties before reliable criteria for the material performance under these special conditions can be established.

The HTGR can be utilized for electricity generation through steam raising or direct-cycle gas-turbine systems and for process heat applications. The levels of helium coolant impurities are expected to be different for the different systems adopted for the HTGR. One of the major differences lies in the oxygen potential (or oxygen partial pressure) of the environment. In the case of a steam-raising system where continuous ingress of low levels of water and steam into the primary coolant is possible, the environment is expected to have a relatively high oxygen potential (i.e., low ratios of $\mathrm{H}_{2}$ to $\mathrm{H}_{2} 0$ ). On the other hand, 
it is anticipated that the helium coolant in the direct-cycle gas-turbine or process heat systems will be much "drier" (i.e., high ratios of $\mathrm{H}_{2}$ to $\mathrm{H}_{2} \mathrm{O}$ ) due to the lack of potential sources of continuous water and steam ingress. This paper reports the results obtained from corrosion tests in the helium environments which are characteristic of both steamraising and gas-turbine/process heat systems. It is the intent of this paper to integrate these results and offer some insights on the mechanistic and kinetic aspects of the corrosion with particular emphasis on carburization in these high-temperature gaseous environments.

The alloys investigated were Alloy $800 \mathrm{H}$, Inconel ${ }^{*}$ Alloy 617 and Hastelloy ${ }^{* *}$ Alloy $X$. These alloys are being considered as candidate materials for the construction of many high-temperature structural components of HTGRs.

\footnotetext{
*Inconel is a registered trademark of the International Nickel Company, Inc. $* *$ Hastelloy is a registered trademark of Cabot Corporation.
} 


\section{MATERIALS AND EXPERIMENTS}

Corrosion tests were conducted in four different helium environments: A, B, C and D. Concentrations of the gaseous impurities in each environment are shown in Table 1. Each test environment was generated by first purifying high-purity nuclear-grade helium and then adding controlled quantities of $\mathrm{H}_{2}, \mathrm{CH}_{4}, \mathrm{CO}, \mathrm{CO}_{2}$ and $\mathrm{H}_{2} \mathrm{O}$ to the purified he1ium.

Purification of the helium for Environments A, B and C (oncethrough systems) was accomplished by passing commercial-purity helium through activated charcoal and/or molecular sieve ( $5 \AA \mathrm{A}$ diam) at liquid nitrogen temperature, while the initial helium supply for Environment D (recirculating loop) was provided by means of a special bypass purification section as described in Ref. 6. The $\mathrm{H}_{2}, \mathrm{CH}_{4}, \mathrm{CO}$ and $\mathrm{CO}_{2}$ concentrations for Environments $\mathrm{A}, \mathrm{B}$ and $\mathrm{C}$ were established by adding controlled quantities of research-purity gases to the purified helium by means of low-flow vacuum leak valves. $\mathrm{H}_{2} \mathrm{O}$ was added to Environments $A$ and $B$ by a bypass flow through hydrated oxalic acid crystals at room temperature and at a controlled flow rate, which was calibrated to yield the desired moisture level. No $\mathrm{H}_{2} \mathrm{O}$ was deliberately added to Environment $\mathrm{C}$ and no $\mathrm{CO}_{2}$ was added to Environments $\mathrm{A}$ and $\mathrm{C}$.

The $\mathrm{H}_{2}, \mathrm{CO}$ and $\mathrm{H}_{2} \mathrm{O}$ contents for the recirculating loop (Environment D) were regulated by varying the amount of $\mathrm{H}_{2} \mathrm{O}$ injected into the loop as well as the temperature and gas flow through a graphite furnace, where $\mathrm{H}_{2} \mathrm{O}$ was converted to $\mathrm{CO}$ and $\mathrm{H}_{2} \cdot \mathrm{CH}_{4}$ was injected into the loop independently. No $\mathrm{CO}_{2}$ was deliberately added to this environment. 
After obtaining the desired concentrations of gaseous impurities, the gas mixtures were introduced into high-temperature retorts where the corrosion tests were conducted. For tests conducted in A, B and C environments, the test gas after passing through the retort was exhausted to the atmosphere. The once-through test systems used for conducting tests in the $A$ and $B$ environments and in the $C$ environment are described in detail in Ref. 1 and Ref. 2, respectively. The test facility (CIIR ${ }^{*}$, a recirculating loop) for Environment D tests is described fully in Ref. 6. All test systems were leak tight with no detectable oxygen (by means of gas chromatography) in the system. The test gas before and after passing through each retort was analyzed by a gas chromatograph for $\mathrm{H}_{2}$ (except for Environment D), $\mathrm{CO}, \mathrm{CH}_{4}$ and $\mathrm{CO}_{2}$. The $\mathrm{H}_{2}$ level for the circulating loop was monitored by converting it to $\mathrm{H}_{2} \mathrm{O}$ in a $\mathrm{Cu} 0$ furnace and employing a $\mathrm{P}_{2} \mathrm{O}_{5}$ Meeco Model $\mathrm{W}$ electrolytic $\mathrm{H}_{2} \mathrm{O}$ analyzer. $\mathrm{H}_{2} \mathrm{O}$ for Environments $\mathrm{A}, \mathrm{B}$ and $\mathrm{C}$ was measured using an EG\&G dew point hygrometer, while $\mathrm{H}_{2} \mathrm{O}$ for Environment $\mathrm{D}$ was monitored with a Goldsmith $\mathrm{P}_{2} \mathrm{O}_{5}$ cell with extra-fine platinum electrodes. Flow rates for the once-through and recirculating loop systems were maintained, depending on the specific system (Table 1), at 180 to $1000 \mathrm{~cm}^{3} / \mathrm{min}$ such that no significant changes or depletion in the concentration of each gas compound were observed between inlet and exit streams of the test retort. Variations of the above impurities during test were generally $\pm 10 \%$ of the nominal.

Alloys studied in the present investigation included Alloy $800 \mathrm{H}$, Inconel Alloy 617 and Hastelloy Alloy $X$. The alloy heat numbers, material forms, grain sizes and specimen configurations for each test environment are shown in Table 2. The chemical compositions of the alloys are tabulated in Table 3.

All specimen surfaces were in the as-machined condition (16 rms or better). The specimens were exposed isothermally at the temperatures given in Table 1. Exposure times were up to $10,000 \mathrm{~h}$.

${ }^{*}$ Central Institute for Industrial Research, Oslo, Norway. 
TABLE 1

EXPOSURE CONDITIONS FOR THE ALLOYS UNDER INVESTIGATION

\begin{tabular}{|c|c|c|c|c|c|c|}
\hline \multirow{2}{*}{$\begin{array}{c}\text { Test } \\
\text { Environment }\end{array}$} & \multicolumn{5}{|c|}{ Gaseous Impurities in Helium ( $\mu \mathrm{atm}$ ) } & \multirow{2}{*}{$\begin{array}{c}\text { Exposure Temperature } \\
{\left[{ }^{\circ} \mathrm{C}\left({ }^{\circ} \mathrm{F}\right)\right]}\end{array}$} \\
\hline & $\mathrm{H}_{2}$ & $\mathrm{CO}$ & $\mathrm{CH}_{4}$ & $\mathrm{H}_{2} \mathrm{O}$ & $\mathrm{CO}_{2}$ & \\
\hline$A^{(a)}$ & 1500 & 450 & 50 & 50 & $<0.5^{(\mathrm{e})}$ & $\begin{array}{l}649,760,871 \\
(1200,1400,1600)\end{array}$ \\
\hline$B^{(b)}$ & 200 & 100 & 20 & 50 & 5 & $\begin{array}{l}649,760,871,900 \\
(1200,1400,1600,1650)\end{array}$ \\
\hline $\mathrm{C}^{(\mathrm{c})}$ & 500 & 50 & 50 & $<0.5^{(\mathrm{e})}$ & $<0.5^{(\mathrm{e})}$ & $\begin{array}{l}800,900 \\
(1472,1650)\end{array}$ \\
\hline$D^{(d)}$ & 500 & 50 & 50 & 1.5 & $<0.5^{(\mathrm{e})}$ & $\begin{array}{l}650,800,900,1000 \\
(1202,1472,1650, \\
1832)\end{array}$ \\
\hline
\end{tabular}

(a) 1.5-atm helium pressure at a flow rate of $\sim 180 \mathrm{~cm}^{3} / \mathrm{min}$ flowing through the test retort $(5.08-\mathrm{cm}-\mathrm{diam}$ alumina retort).

(b) 1.5 -atm helium pressure at a flow rate of $\sim 300 \mathrm{~cm}^{3} / \mathrm{min}$ flowing through the test retort $(5.08-\mathrm{cm}-\mathrm{diam}$ alumina retort $)$.

(c) 1.05-atm helium pressure at a flow rate of $2500 \mathrm{~cm}^{3} / \mathrm{min}$ flowing through the test retort (17.1-cm-diam Inconel Alloy 600 retort).

(d) 1.7-atm helium pressure at a flow rate of $\sim 1000 \mathrm{~cm}^{3} / \mathrm{min}$ flowing through the test retort $(7.9-\mathrm{cm}-\mathrm{diam}$ Inconel Alloy 600 retort).

${ }^{(e)}$ Concentration was below the detection 1imit of the instrument

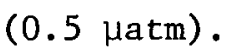


TABLE 2

HEAT NUMBER, FORM ANND GRAIN SIZE OF ALLOYS TESTED IN EACH ENVIRONMENT

\begin{tabular}{|c|c|c|c|c|}
\hline $\begin{array}{l}\text { Test } \\
\text { Environment }\end{array}$ & Material & Heat Number & $\begin{array}{l}\text { Material Form } \\
\text { (Specimen Size) }\end{array}$ & $\begin{array}{l}\text { ASTM } \\
\text { Grain } \\
\text { Size }\end{array}$ \\
\hline \multirow[t]{3}{*}{ A } & A11oy $800 \mathrm{H}$ & HH6979A & $\begin{array}{l}1.9-\mathrm{cm} \text {-diam rod } \\
(0.15 \times 1.9 \mathrm{~cm} \text { diam })\end{array}$ & 4 \\
\hline & Inconel Alloy 617 & XX00A7US & $\begin{array}{l}0.12-\mathrm{cm}-\text { thick sheet } \\
(0.12 \times 1.5 \times 2 \mathrm{~cm})\end{array}$ & 5 \\
\hline & Haste1loy Alloy $\mathrm{X}$ & $2600-1-4547$ & $\begin{array}{l}0.14-\mathrm{cm}-\text { thick sheet } \\
(0.14 \times 1.5 \times 2 \mathrm{~cm})\end{array}$ & 4 \\
\hline \multirow[t]{3}{*}{ B } & Alloy $800 \mathrm{H}$ & HH6993A & $\begin{array}{l}\text { 1.9-cm-diam rod } \\
(0.13 \times 1.25 \times 2.54 \mathrm{~cm})\end{array}$ & 4 \\
\hline & Inconel Alloy 617 & XX00A5US & $\begin{array}{l}0.165-\mathrm{cm}-\mathrm{thick} \text { sheet } \\
(0.13 \times 1.25 \times 2.54 \mathrm{~cm})\end{array}$ & 7 \\
\hline & Hastelloy Alloy $\mathrm{X}$ & $2600-1-4547$ & $\begin{array}{l}0.14-\mathrm{cm} \text {-thick sheet } \\
(0.13 \times 1.25 \times 2.54 \mathrm{~cm})\end{array}$ & 5 \\
\hline \multirow[t]{3}{*}{$\mathrm{C}$} & Inconel Alloy 617 & XX00A4US & $\begin{array}{l}1.27-\mathrm{cm} \text {-diam rod } \\
(0.63-\mathrm{cm}-\text { diam } \times 2.54-\mathrm{cm} \text { length })\end{array}$ & 3 \\
\hline & Haste1loy A1loy X & C15567 & $\begin{array}{l}\text { 1.27-cm-diam rod } \\
\text { (0.63-cm-diam x } 2.54-\mathrm{cm} \text { length) }\end{array}$ & 4 \\
\hline & & $5-2831$ & $\begin{array}{l}\text { 1.27-cm-diam rod } \\
(0.63-\mathrm{cm}-\text { diam } \times 2.54-\mathrm{cm} \text { length })\end{array}$ & 3 \\
\hline \multirow[t]{4}{*}{$\mathrm{D}$} & A11oy $800 \mathrm{H}$ & HH6979A & $\begin{array}{l}\text { 1.9-cm-diam rod } \\
(0.63-\mathrm{cm} \text {-diam } \times 2.54-\mathrm{cm} \text { length })\end{array}$ & 4 \\
\hline & Inconel Alloy 617 & XX00A4US & $\begin{array}{l}1.27-\mathrm{cm} \text {-diam rod } \\
(0.63-\mathrm{cm}-\mathrm{d} \text { iam } \times 2.54-\mathrm{cm} \text { length })\end{array}$ & 3 \\
\hline & Hastelloy Alloy X & C15567 & $\begin{array}{l}1.27-\mathrm{cm} \text {-diam rod } \\
(0.63-\mathrm{cm} \text {-diam } \mathrm{x} 2.54-\mathrm{cm} \text { length })\end{array}$ & 4 \\
\hline & & $5-2831$ & $\begin{array}{l}\text { 1.27-cm-diam rod } \\
(0.63-\mathrm{cm}-\text { diam } \times 2.54-\mathrm{cm} \text { length })\end{array}$ & 3 \\
\hline
\end{tabular}


TABLE 3

CHEMICAL COMPOSITION OF ALLOYS UNDER INVESTIGATION

\begin{tabular}{|c|c|c|c|c|c|c|c|c|c|c|c|c|c|c|}
\hline \multirow[b]{2}{*}{ Material } & \multirow[b]{2}{*}{ Heat Number } & \multicolumn{13}{|c|}{ Chemical Composition (wt \%) (a) } \\
\hline & & $\mathrm{C}$ & $\mathrm{Fe}$ & $\mathrm{N} \mathbf{i}$ & $\mathrm{Cr}$ & Co & Mo & Al & $\mathrm{Ti}$ & $\mathrm{Mn}$ & Si & $\mathrm{s}$ & $P$ & Other \\
\hline \multirow[t]{2}{*}{ Alloy $800 \mathrm{H}$} & HH6979A & 0.07 & 43.6 & 33.4 & 20.4 & -- & -- & 0.43 & 0.46 & 0.77 & 0.39 & 0.004 & -- & $\mathrm{Cu}=0.49$ \\
\hline & HH6993A & 0.07 & 45.9 & 31.7 & 20.1 & -- & -- & 0.25 & 0.35 & 0.80 & 0.32 & 0.006 & -- & $\mathrm{Cu}=0.53$ \\
\hline \multirow[t]{3}{*}{ Inconel Alloy 617} & XX00A7US & 0.08 & 0.5 & 54.5 & 22.3 & 12.45 & 9.13 & 0.89 & -- & 0.03 & 0.16 & 0.007 & -- & \\
\hline & XX00A5US & 0.07 & 0.19 & 55.9 & 21.8 & 12.24 & 8.71 & 0.99 & -- & 0.03 & 0.06 & 0.007 & -- & \\
\hline & $\mathrm{XX00A4US}$ & 0.07 & 0.15 & 54.7 & 22.3 & 12.46 & 9.09 & 1.06 & -- & 0.02 & 0.08 & 0.007 & -- & \\
\hline \multirow[t]{3}{*}{ Hastelloy Alloy $\mathrm{X}$} & $2600-1-4547$ & 0.08 & 18.7 & Bal & 21.3 & 2.03 & 9.44 & -- & -- & 0.61 & 0.32 & 0.004 & 0.003 & $\begin{array}{l}W-0.62 \\
B-\leqslant 0.002\end{array}$ \\
\hline & C15567 & 0.08 & 19.1 & $\mathrm{Bal}$ & 21.5 & 2.11 & 8.95 & -- & -- & 0.71 & 0.53 & 0.005 & 0.018 & $\begin{array}{l}W-0.45 \\
B-0.0029\end{array}$ \\
\hline & & 0.09 & 18.8 & $\mathrm{Ba} 1$ & 21.5 & 2.08 & 8.76 & 0.22 & 0.01 & 0.61 & 0.50 & 0.005 & 0.023 & $\begin{array}{l}W-0.60 \\
C u-0.16 \\
B-<0.002\end{array}$ \\
\hline
\end{tabular}

${ }^{(a)}$ Vendor's analysis. 
Following exposure, the specimens were subjected to a detailed metallurgical examination that included optical metallography, electron microprobe analysis, scanning electron microscopy with energy dispersive analyzer, X-ray diffraction, and bulk carbon analysis. 


\section{EXPERIMENTAL RESULTS}

The results of bulk carbon analysis of specimens exposed to the various environments are presented in Tables 4 through 7 . The results are expressed as mass of bulk carbon increase per unit surface area $\left(\mathrm{g} / \mathrm{cm}^{2}\right)$, which was determined by the relationship

$$
\Delta \mathrm{M}\left(\mathrm{g} / \mathrm{cm}^{2}\right)=\Delta \mathrm{C}\left(\frac{\mathrm{W}}{\mathrm{A}}\right),
$$

where $\Delta M=$ mass of carbon increase $\left(\mathrm{g} / \mathrm{cm}^{2}\right)$,

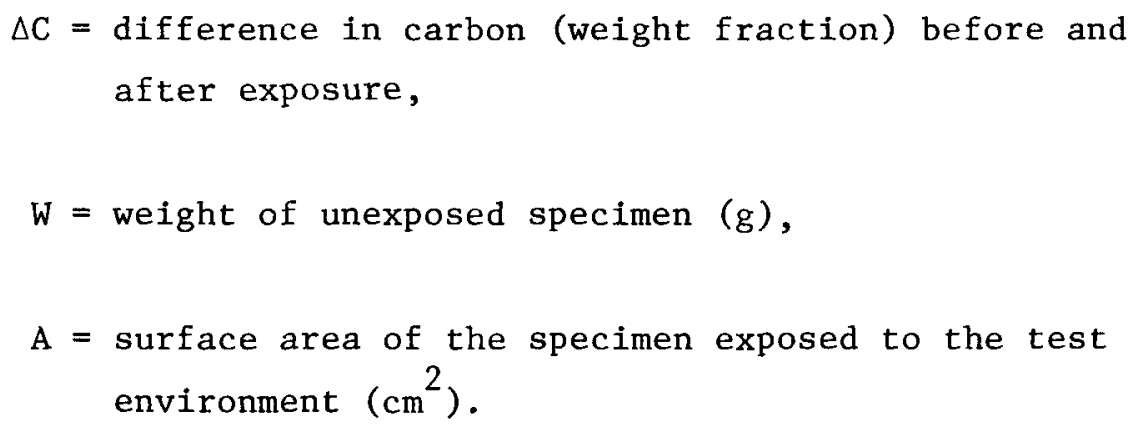

As shown in Tables 4 through 7 , carburization was observed in all four environments. The data presented are somewhat limited for establishing the rate of carburization for each alloy and environment. Nevertheless, an attempt was made to examine the carburization rate in terms of carbon increase as a function of time for Hastelloy Alloy $X$ tested in $C$ and $D$ environments. The gas chemistry of Environment $C$ was essentially the same as Environment $D$ except for a lower moisture leve1. No significant differences in carburization kinetics were observed between these two environments. The same heat of material was used for both environments. Accordingly, the data from both environments were plotted together and are shown in Fig. 1. 
RESULTS OF BULK CARBON ANALYSIS $(a)$ TABLE 4 SPECIMENS EXPOSED IN ENVIRONMENT A

\begin{tabular}{|c|c|c|c|c|c|c|}
\hline Material & Heat Number & $\begin{array}{l}\text { Specimen } \\
\text { Number }\end{array}$ & $\begin{array}{l}\text { Test Temp } \\
{\left[{ }^{\circ} \mathrm{C}\left({ }^{\circ} \mathrm{F}\right)\right]}\end{array}$ & $\begin{array}{c}\text { Test } \\
\text { T ime } \\
\text { (h) }\end{array}$ & $\begin{array}{l}\text { Percent of } \\
\text { Carbon Increase } \\
\quad(\text { wt \%) }(b)\end{array}$ & $\begin{array}{c}\text { Mass of } \\
\text { Carbon Increase } \\
\left(\mathrm{g} / \mathrm{cm}^{2}\right)(\mathrm{c})\end{array}$ \\
\hline \multirow[t]{4}{*}{ Alloy $800 \mathrm{H}$} & \multirow[t]{4}{*}{ НH6979A } & $4-7$ & $649(1200)$ & 5,000 & 0 & 0 \\
\hline & & $5-7$ & $760(1400)$ & 5,000 & 0.001 & $5.8 \times 10^{-6}$ \\
\hline & & $8-6-1$ & 871 (1600) & 2,000 & 0.009 & $5.23 \times 10^{-5}$ \\
\hline & & $8-6$ & $871(1600)$ & 5,000 & 0.010 & $5.8 \times 10^{-5}$ \\
\hline \multirow{4}{*}{$\begin{array}{l}\text { Inconel Alloy } 617 \\
\text { Hastelloy Alloy X }\end{array}$} & \multirow{4}{*}{$\begin{array}{l}\text { XX00A7US } \\
2600-1-4547\end{array}$} & $5-5$ & $760(1400)$ & 5,000 & 0 & 0 \\
\hline & & $4-2$ & $649(1200)$ & 5,000 & 0.002 & $1.15 \times 10^{-5}$ \\
\hline & & $5-2$ & $760(1400)$ & 5,000 & 0 & 0 \\
\hline & & $6-2$ & $871(1600)$ & 5,000 & 0.007 & $4.0 \times 10^{-5}$ \\
\hline
\end{tabular}

(a) One measurement per specimen.

(b) The difference in bulk carbon content (wt \%) before and after exposure.

(c) Mass of carbon increase per unit surface area of the specimen after exposure to the environment. 
TABLE 5

RESULTS OF BULK CARBON ANALYSIS ${ }^{(a)}$ ON SPECIMENS EXPOSED IN ENVIRONMENT B

\begin{tabular}{|c|c|c|c|c|c|c|c|}
\hline Material & Heat Number & $\begin{array}{l}\text { Specimen } \\
\text { Number }\end{array}$ & $\begin{array}{l}\text { Test } \\
1^{\circ} \mathrm{C}\end{array}$ & $\begin{array}{l}\text { Temp } \\
\left.\left({ }^{\circ} \mathrm{F}\right)\right]\end{array}$ & $\begin{array}{l}\text { Test } \\
\text { Time } \\
\text { (hr) }\end{array}$ & $\begin{array}{l}\text { Percent of } \\
\text { Carbon Increase } \\
\text { (wt } \%)(b)\end{array}$ & $\begin{array}{c}\text { Mass of } \\
\text { Carbon Increase } \\
\left(\mathrm{g} / \mathrm{cm}^{2}\right)(\mathrm{c})\end{array}$ \\
\hline \multirow[t]{8}{*}{ Alloy $800 \mathrm{H}$} & \multirow[t]{8}{*}{ HH6993A } & HD 2 & 649 & $(1200)$ & 5000 & 0.002 & $1.0 \times 10^{-5}$ \\
\hline & & HD4 & 649 & $(1200)$ & 7000 & 0.01 & $5.06 \times 10^{-5}$ \\
\hline & & HE2 & 760 & $(1400)$ & 5000 & 0.008 & $3.98 \times 10^{-5}$ \\
\hline & & $\mathrm{HE} 4$ & 760 & $(1400)$ & 7000 & 0.014 & $7.0 \times 10^{-5}$ \\
\hline & & $\mathrm{HF} 2$ & 871 & $(1600)$ & 5000 & 0.05 & $2.5 \times 10^{-4}$ \\
\hline & & $\mathrm{HF}_{4}$ & 871 & $(1600)$ & 7000 & 0.048 & $2.38 \times 10^{-4}$ \\
\hline & & $\mathrm{HG} 2$ & 900 & $(1650)$ & 5000 & 0.025 & $1.28 \times 10^{-4}$ \\
\hline & & HG4 & 900 & (1650) & 7000 & 0.028 & $1.4 \times 10^{-4}$ \\
\hline \multirow[t]{8}{*}{ Incone1 Alloy 617} & \multirow[t]{8}{*}{ XX00A5US } & $7 \mathrm{D} 2$ & 649 & $(1200)$ & 5000 & 0.003 & $1.69 \times 10^{-5}$ \\
\hline & & $7 \mathrm{D} 4$ & 649 & $(1200)$ & 7000 & 0.0085 & $4.7 \times 10^{-5}$ \\
\hline & & $7 \mathrm{E} 2$ & 760 & $(1400)$ & 5000 & 0.007 & $3.85 \times 10^{-5}$ \\
\hline & & $7 \mathrm{E} 4$ & 760 & $(1400)$ & 7000 & 0.009 & $4.85 \times 10^{-5}$ \\
\hline & & $7 F 2$ & 871 & $(1600)$ & 5000 & 0.025 & $1.4 \times 10^{-4}$ \\
\hline & & $7 F 4$ & 871 & $(1600)$ & 7000 & 0.0265 & $1.43 \times 10^{-4}$ \\
\hline & & $7 \mathrm{G} 2$ & 900 & $(1650)$ & 5000 & 0.011 & $5.96 \times 10^{-5}$ \\
\hline & & $7 \mathrm{G} 4$ & 900 & (1650) & 7000 & 0.024 & $1.32 \times 10^{-4}$ \\
\hline \multirow[t]{8}{*}{ Hastelloy Alloy X } & \multirow[t]{8}{*}{$2600-1-4547$} & $\mathrm{XD} 2$ & 649 & $(1200)$ & 5000 & 0.004 & $2.17 \times 10^{-5}$ \\
\hline & & $\mathrm{XD} 4$ & 649 & $(1200)$ & 7000 & 0.012 & $6.53 \times 10^{-5}$ \\
\hline & & $\mathrm{XE} 2$ & 760 & $(1400)$ & 5000 & 0.006 & $3.36 \times 10^{-5}$ \\
\hline & & XF4 & 760 & $(1400)$ & 7000 & 0.015 & $8.24 \times 10^{-5}$ \\
\hline & & $\mathrm{XF} 2$ & 871 & $(1600)$ & 5000 & 0.025 & $1.38 \times 10^{-4}$ \\
\hline & & $\mathrm{XE} 4$ & 871 & $(1600)$ & 7000 & 0.013 & $6.98 \times 10^{-5}$ \\
\hline & & $\mathrm{XG} 2$ & 900 & (1650) & 5000 & 0.023 & $1.27 \times 10^{-4}$ \\
\hline & & XG4 & 900 & (1650) & 7000 & 0.042 & $2.28 \times 10^{-4}$ \\
\hline
\end{tabular}

(a) Two measurements per specimen.

(b) The difference in bulk carbon content (wt \%) before and after exposure.

(c) Mass of carbon increase per unit surface area of the specimen after exposure to the environment. 
TABLE 6

RESULTS OF BULK CARBON ANALYSIS ON SPECIMENS EXPOSED IN ENVIRONMENT C

\begin{tabular}{|c|c|c|c|c|c|c|}
\hline Material & $\begin{array}{l}\text { Heat } \\
\text { Number }\end{array}$ & $\begin{array}{l}\text { Specimen } \\
\text { Number }\end{array}$ & $\begin{array}{l}\text { Test Temp } \\
{\left[{ }^{\circ} \mathrm{C}\left({ }^{\circ} \mathrm{F}\right)\right]}\end{array}$ & $\begin{array}{l}\text { Test } \\
\text { Time } \\
\text { (hr) }\end{array}$ & $\begin{array}{l}\text { Percent of } \\
\text { Carbon Increase } \\
\text { (wt } \%)(a)\end{array}$ & $\begin{array}{c}\text { Mass of } \\
\text { Carbon Increase } \\
\left(\mathrm{g} / \mathrm{cm}^{2}\right)(\mathrm{b})\end{array}$ \\
\hline \multirow[t]{5}{*}{ Inconel Alloy 617} & \multirow[t]{5}{*}{ XX00A4US } & $09 i 56$ & $800(1472)$ & 3000 & 0.011 & $1.37 \times 10^{-4}$ \\
\hline & & 09157 & $800(1472)$ & 7000 & 0.014 & $1.74 \times 10^{-4}$ \\
\hline & & 09158 & $800(1472)$ & 10000 & $0.0175^{(\mathrm{c})}$ & $2.19 \times 10^{-4}$ \\
\hline & & $09142 \mathrm{~A}$ & $900(1650)$ & 3000 & $0.051^{(c)}$ & $6.30 \times 10^{-4}$ \\
\hline & & 09178 & $900(1650)$ & 7000 & $0.076^{(c)}$ & $4.05 \times 10^{-4}$ \\
\hline \multirow[t]{18}{*}{ Hastelloy A1loy X } & \multirow[t]{11}{*}{ C15567 } & 08161 & $800(1472)$ & 3000 & 0.018 & $2.22 \times 10^{-4}$ \\
\hline & & 08115 & $800(1472)$ & 3000 & 0.018 & $2.24 \times 10^{-4}$ \\
\hline & & 08116 & $800(1472)$ & 3000 & 0.018 & $2.24 \times 10^{-4}$ \\
\hline & & 08182 & $800(1472)$ & 3000 & 0.028 & $4.92 \times 10^{-4}$ \\
\hline & & 08162 & $800(1472)$ & 7000 & 0.018 & $2.22 \times 10^{-4}$ \\
\hline & & 08163 & $800(1472)$ & 10000 & 0.028 & $3.41 \times 10^{-4}$ \\
\hline & & 08125 & $900(1650)$ & 3000 & 0.078 & $9.60 \times 10^{-4}$ \\
\hline & & 08126 & $900(1650)$ & 3000 & 0.068 & $8.37 \times 10^{-4}$ \\
\hline & & 08184 & $900(1650)$ & 3000 & 0.068 & $1.19 \times 10^{-3}$ \\
\hline & & $08142 \mathrm{~A}$ & $900(1650)$ & 3000 & $0.038^{(c)}$ & $4.66 \times 10^{-4}$ \\
\hline & & $08143 \mathrm{~A}$ & $900(1650)$ & 7000 & 0.118 & $1.45 \times 10^{-3}$ \\
\hline & \multirow[t]{7}{*}{$5-2831$} & 08607 & $800(1472)$ & 3000 & 0.017 & $2.09 \times 10^{-4}$ \\
\hline & & 08683 & $800(1472)$ & 3000 & 0.017 & $2.87 \times 10^{-4}$ \\
\hline & & 08684 & $800(1472)$ & 3000 & 0.015 & $2.52 \times 10^{-4}$ \\
\hline & & 08653 & $800(1472)$ & 3000 & 0.010 & $1.23 \times 10^{-4}$ \\
\hline & & 08602 & $900(1650)$ & 3000 & 0.05 & $6.15 \times 10^{-4}$ \\
\hline & & 08604 & $900(1650)$ & 3000 & 0.10 & $1.24 \times 10^{-3}$ \\
\hline & & 08643 & $900(1650)$ & 3000 & 0.06 & $7.41 \times 10^{-4}$ \\
\hline
\end{tabular}

(a) The difference in bulk carbon content (wt \%) before and after exposure.

(b) Mass of carbon increase per unit surface area of the specimen after exposure to the environment.

(c) Average of two measurements per specimens, others one measurement per specimen. 
TABLE 7

RESULTS OF BULK CARBON ANALYSIS ON SPECIMENS EXPOSED IN ENVIRONMENT D

\begin{tabular}{|c|c|c|c|c|c|c|}
\hline Material & Heat Number & $\begin{array}{l}\text { Spec1men } \\
\text { Number }\end{array}$ & $\begin{array}{l}\text { Test Temp } \\
{\left[{ }^{\circ} \mathrm{C}\left({ }^{\circ} \mathrm{F}\right)\right]}\end{array}$ & $\begin{array}{l}\text { Test } \\
\text { Time } \\
\text { (hr) }\end{array}$ & $\begin{array}{l}\text { Percent of } \\
\text { Carbon Increase } \\
(w t \%)(a)\end{array}$ & $\begin{array}{c}\text { Mass of } \\
\text { Carbon Increase } \\
\left(\mathrm{g} / \mathrm{cm}^{2}\right)(\mathrm{b})\end{array}$ \\
\hline \multirow[t]{3}{*}{ Alloy $800 \mathrm{H}$} & \multirow[t]{3}{*}{ HH6979A } & 40141 & $899(1650)$ & 8,700 & 0.08 & $9.48 \times 10^{-4}$ \\
\hline & & 40142 & $900(1650)$ & 3,000 & 0.06 & $7.11 \times 10^{-4}$ \\
\hline & & 40143 & $1000(1832)$ & 3,000 & 0.07 & $8.31 \times 10^{-4}$ \\
\hline \multirow[t]{6}{*}{ Inconel Alloy 617} & \multirow[t]{6}{*}{ XX00A4US } & 09145 & $650(1202)$ & 3,000 & 0.007 & $8.73 \times 10^{-5}$ \\
\hline & & 09146 & $650(1202)$ & 10,000 & $0.045^{(c)}$ & $5.60 \times 10^{-5}$ \\
\hline & & 09141 & $800(1472)$ & 3,000 & 0.011 & $1.36 \times 10^{-4}$ \\
\hline & & 09142 & $800(1472)$ & 10,000 & 0.008 & $9.99 \times 10^{-5}$ \\
\hline & & 09143 & $900(1650)$ & 3,000 & 0.096 & $1.19 \times 10^{-3}$ \\
\hline & & 09144 & $900(1650)$ & 10,000 & 0.206 & $2.53 \times 10^{-3}$ \\
\hline \multirow[t]{12}{*}{ Hastelloy Alloy X } & \multirow[t]{6}{*}{ C15567 } & 08145 & $650(1202)$ & 3,000 & 0.008 & $9.96 \times 10^{-5}$ \\
\hline & & 08146 & $650(1202)$ & 10,000 & 0.008 & $9.96 \times 10^{-5}$ \\
\hline & & 08141 & $800(1472)$ & 3,000 & 0.028 & $3.45 \times 10^{-4}$ \\
\hline & & 08142 & $800(1472)$ & 10,000 & 0.068 & $8.40 \times 10^{-4}$ \\
\hline & & 08143 & $900(1650)$ & 3,000 & 0.058 & $7.17 \times 10^{-4}$ \\
\hline & & 08144 & $900(1650)$ & 10,000 & $0.168^{(c)}$ & $2.08 \times 10^{-3}$ \\
\hline & \multirow[t]{6}{*}{$5-2831$} & 08645 & $800(1472)$ & 3,000 & 0.015 & $1.84 \times 10^{-4}$ \\
\hline & & 08646 & $800(1472)$ & 10,000 & 0.03 & $3.70 \times 10^{-4}$ \\
\hline & & 08647 & $900(1650)$ & 3,000 & 0.08 & $9.66 \times 10^{-4}$ \\
\hline & & 08648 & $900(1650)$ & 10,000 & 0.15 & $1.84 \times 10^{-3}$ \\
\hline & & 08649 & $1000(1832)$ & 3,000 & 0.11 & $1.35 \times 10^{-3}$ \\
\hline & & 08650 & $1000(1832)$ & 10,000 & 0.221 & $2.73 \times 10^{-3}$ \\
\hline
\end{tabular}

(a) The difference in bulk carbon content (wt \%) before and after exposure.

(b) Mass of carbon increase per unit surface area of the specimen after exposure to the environment.

(c) Average of two measurements per specimen, others one measurement per specimen. 


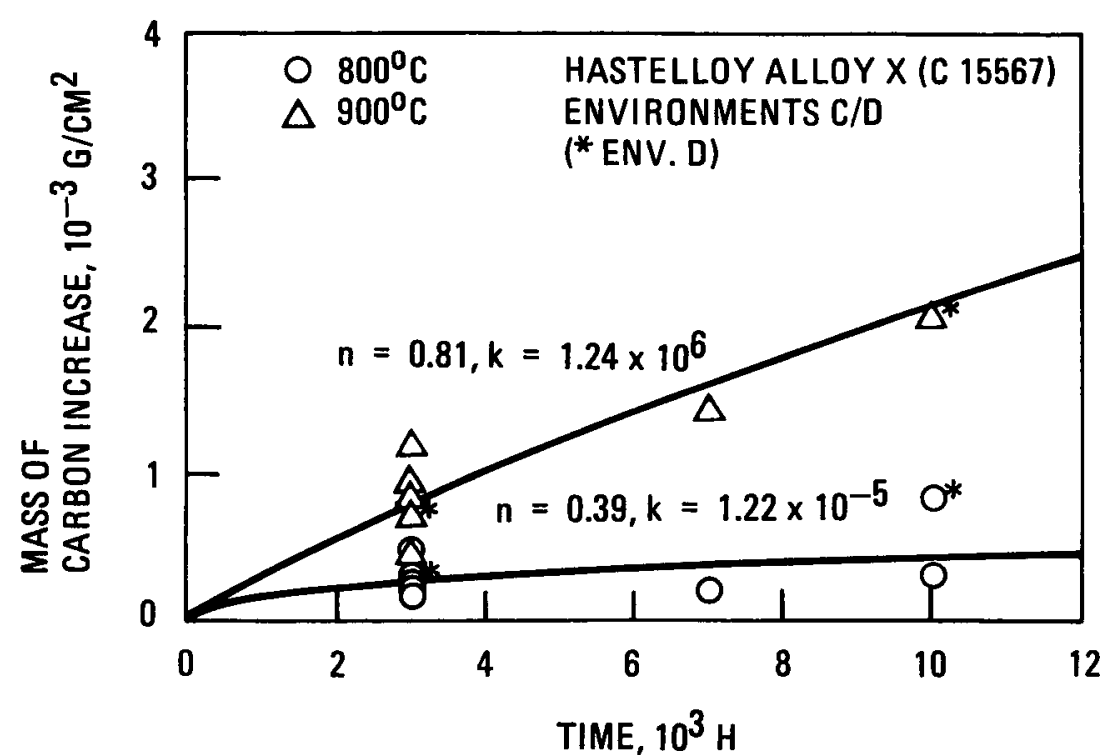

(a)

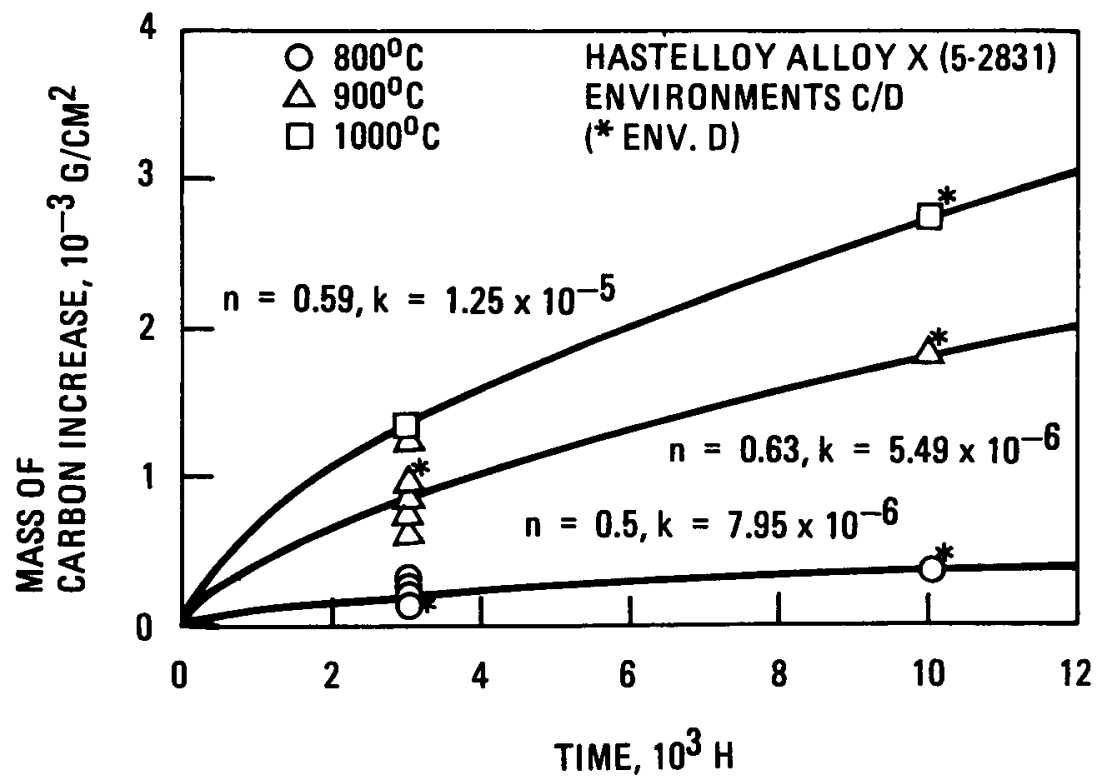

(b)

Fig. 1. Mass of carbon increase as a result of exposure in Environments C/D at various temperatures and times for Hastelloy Alloy X: (a) Heat C15567, (b) Heat 5-2831. 
It is assumed, as a first approximation, that carburization of this a1loy follows a law of the type $\Delta M=k t^{n}$, where $\Delta M$ is the mass of carbon increase per unit surface area, $t$ is the exposure time, and $k$ and $n$ are constants. Both $\mathrm{n}$ and $\mathrm{k}$ were determined by a least-squares fit. Values of the rate exponent ( $n$ ) are slightly less than or about 0.5 (the theoretical value for a parabolic rate) at $800^{\circ} \mathrm{C}\left(1472^{\circ} \mathrm{F}\right)$ and higher than 0.5 (between 0.5 and 1.0 ) at higher temperatures [e.g., $900^{\circ}$ and $1000^{\circ} \mathrm{C}$ $\left(1650^{\circ}\right.$ and $\left.\left.1832^{\circ} \mathrm{F}\right)\right]$. Obviously, more data are needed before the carburization rate constants in these environments can be quantitatively established.

Nevertheless, a significant observation was made in the present investigation. In examining the kinetic data generated for the four environments, it was found that the rates of carburization in high-oxygen potential environments ( $A$ and $B$ ) were significantly lower than those of low-oxygen potential environments (C and D). This difference became more pronounced at higher temperatures [e.g., $900^{\circ}$ and $1000^{\circ} \mathrm{C}\left(1650^{\circ}\right.$ and $\left.1832^{\circ} \mathrm{F}\right)$ ], regardless of whether rates were calculated by a parabolic or a linear rate law. For the purpose of illustrating this observation, carburization rates for all four environments were calculated using the parabolic law and are shown in Figs. 2 through 4 for the three alloys. The data for the $C$ and $D$ environments and those for the $A$ and $B$ environments can be grouped into two separate sets. Clearly, the rates obtained from low-oxygen potential environments ( $C$ and $D$ ) were significantly higher than those of high-oxygen potential environments ( $A$ and $B$ ) for all three alloys.

It might be argued that the heats of material used in $C$ and $D$ environments were different from those used in $A$ and $B$ environments, suggesting that the differences in carburization rates could be attributed to heat-to-heat variations. However, specimens of Alloy $800 \mathrm{H}$ tested in both $A$ and $D$ environments were from the same heat of material. Carburization rates observed in Environment D (low-oxygen potential) were an 


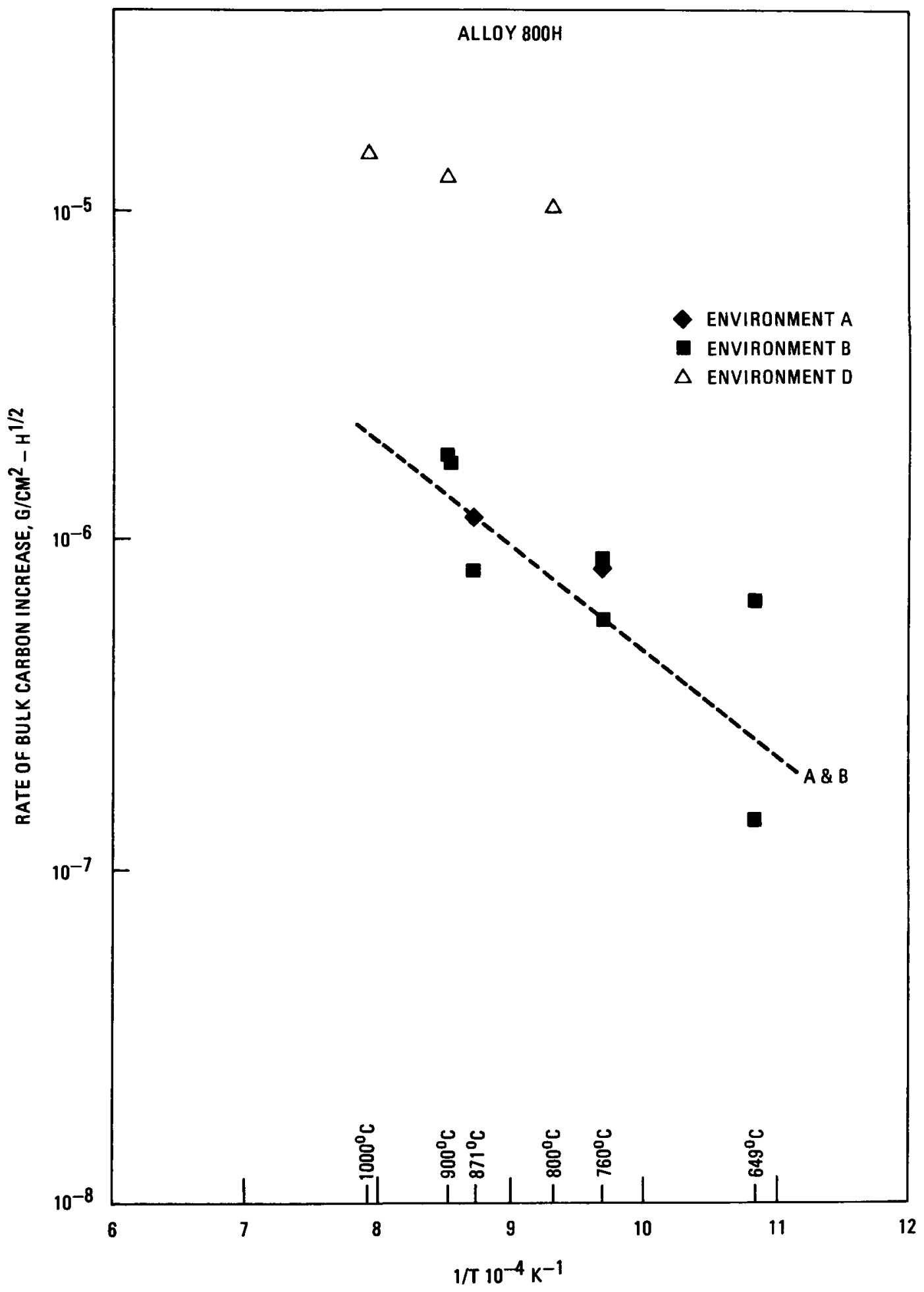

Fig. 2. Rates of carbon increase in Alloy $800 \mathrm{H}$ as a result of exposure to Environments A, B and D as a function of inverse of exposure temperatures (absolute temperatures). 


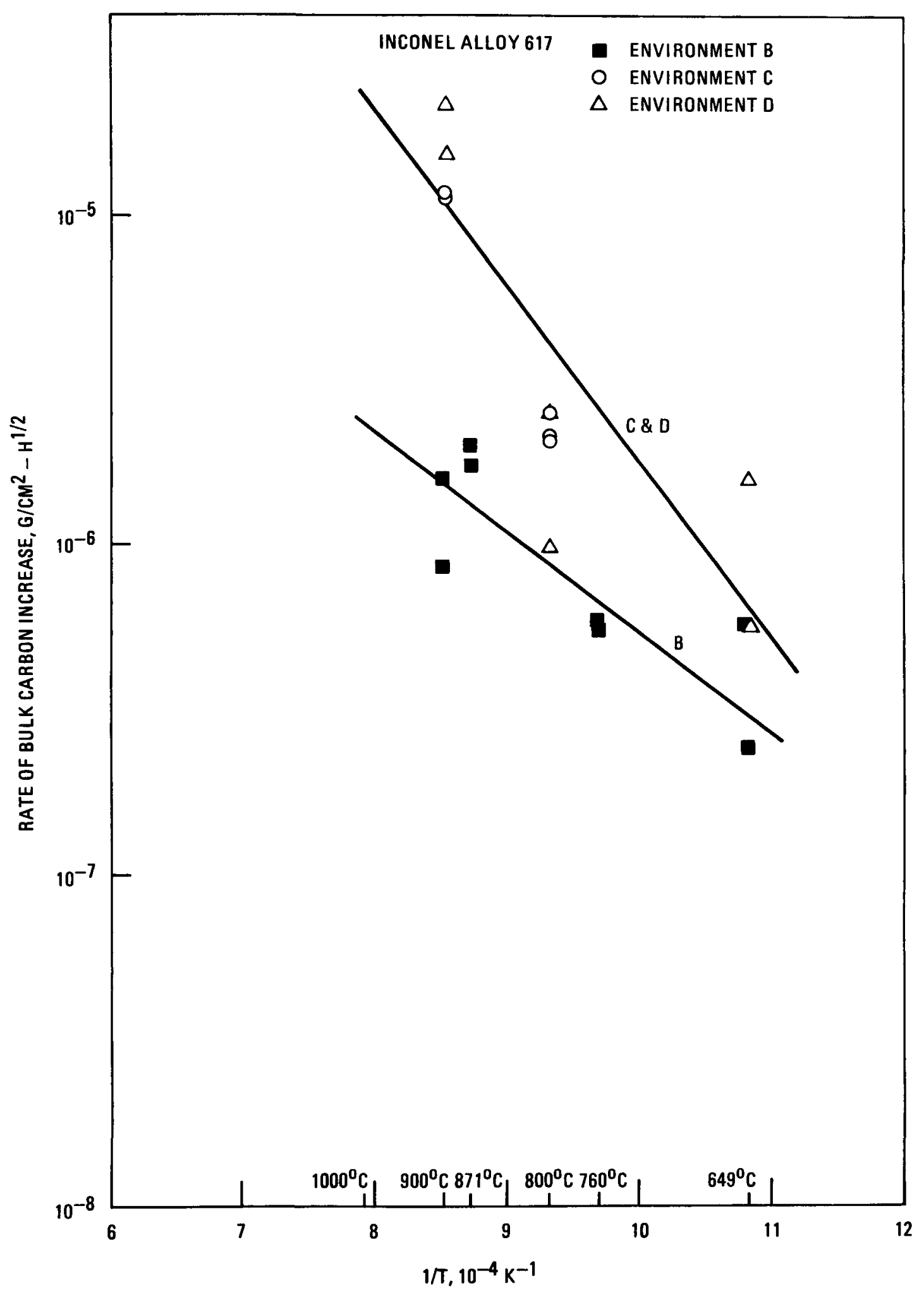

Fig. 3. Rates of carbon increase in Inconel Alloy 617 as a result of exposure to Environments $B, C$ and $D$ as a function of inverse of exposure temperatures (absolute temperatures). 


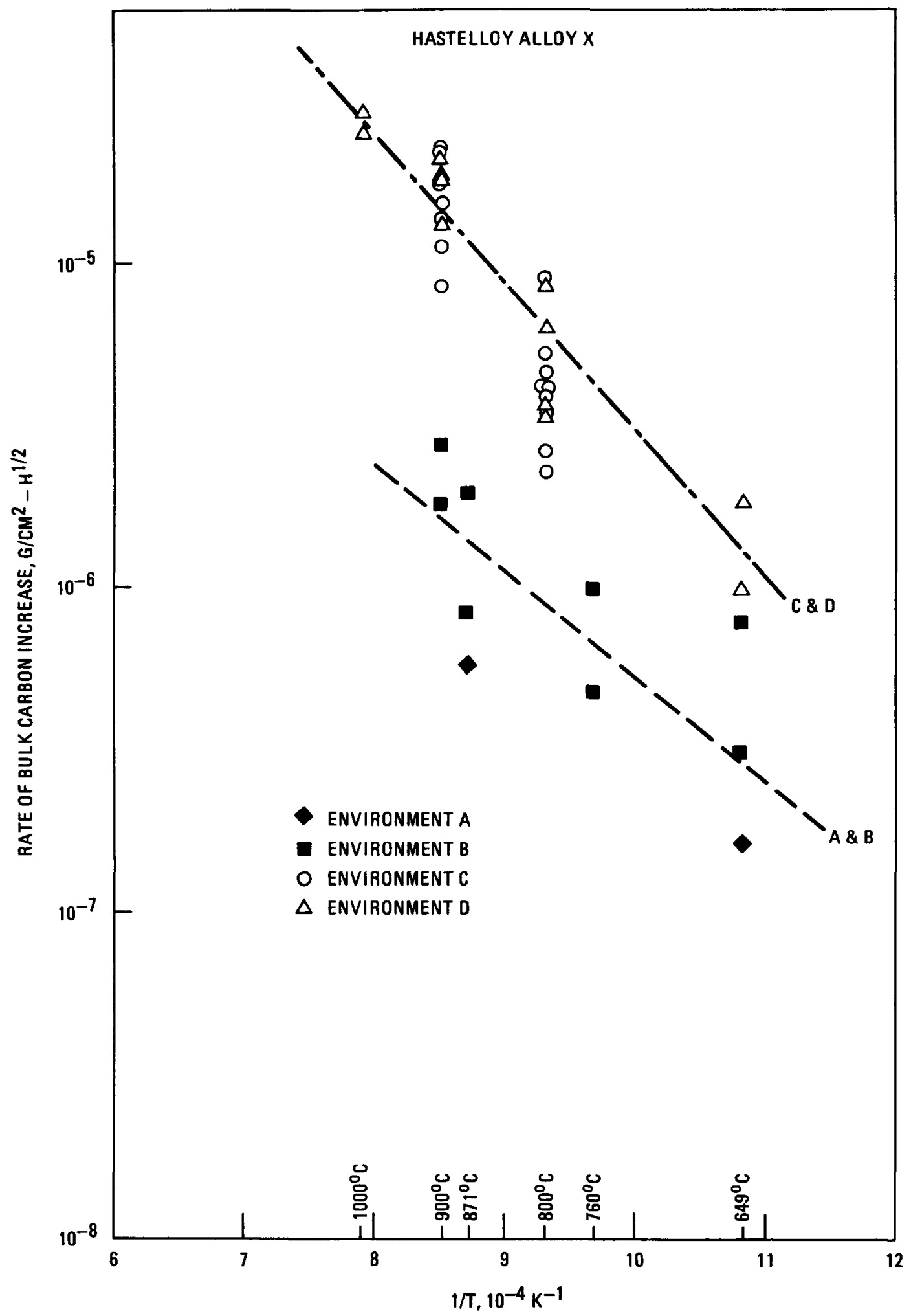

Fig. 4. Rates of carbon increase in Hastelloy Alloy $X$ as a result of exposure to Environments $A, B, C$ and $D$ as a function of inverse of exposure temperatures (absolute temperatures). 
order of magnitude higher than those in Environment A (high-oxygen potential), as illustrated in Fig. 2. Also, two heats of Hastelloy Alloy $X$ were tested in $C$ and $D$ environments, and carburization rates were found to be quite similar. The kinetic data obtained by Inouye (Ref. 7) using different heats of Alloy $800 \mathrm{H}$, Inconel Alloy 617 and Hastelloy Alloy X, tested in an environment similar to $C$ and $D$ environments, were comparable to those obtained in $C$ and $D$ environments in the present study. Tests conducted on the same heat of Hastelloy Alloy $X$ in both $A$ and $B$ environments revealed no significant differences in carburization kinetics (Fig. 4). It is therefore believed that the differences in carburization kinetics observed between the "wet" (A and B) and "dry" (C and D) environments are related to the relative oxygen potentials for the two types of environments. 


\section{DISCUSSION}

The helium coolant in an operating reactor generally makes a complete circuit (viz., from the core to the heat exchangers or gas turbines and back to the core) in several seconds. The gas components in the coolant do not react with each other to reach an equilibrium state under this dynamic condition. Thus, the state of the gas mixtures in the helium coolant is a nonequilibrium condition. In order to simulate this nonequilibrium state of the gas mixtures in the helium coolant, the flow rate of the test gas employed in the tests, although not reaching the reactor flow rate, is normal1y fast enough to maintain a nonequilibrium state, i.e., no depletion of each gas component during passage through the test retort. This was checked by gas analysis at the inlet and exit streams of the test retort.

Since the gas mixtures in the environment are in a nonequilibrium state, the gas-metal reaction can be approximated by considering each individual chemical reaction interacting with the metal. Carburization of metals by the gas mixtures is possible by one or more of the following reactions:

$$
\begin{aligned}
& \mathrm{CO}+\mathrm{H}_{2} \rightleftharpoons \mathrm{C}+\mathrm{H}_{2} \mathrm{O} \\
& 2 \mathrm{CO} \rightleftharpoons \mathrm{C}+\mathrm{CO}_{2} \\
& \mathrm{CH}_{4} \rightleftharpoons \mathrm{C}+2 \mathrm{H}_{2} \\
& \mathrm{CH}_{4}+\mathrm{CO} \rightleftharpoons 2 \mathrm{C}+\mathrm{H}_{2} \mathrm{O}+\mathrm{H}_{2}
\end{aligned}
$$

The carbon activity in the gas phase for each of the four reactions can be calculated by its equilibrium constant (k):

$$
a_{c}=k \frac{{ }_{C O} \cdot{ }^{P_{H_{2}}}}{{ }_{\mathrm{P}_{2} \mathrm{O}}} \text {, for Reaction }
$$




$$
\begin{aligned}
& a_{c}=k_{2} \frac{\left(P_{C O}\right)^{2}}{{ }_{\mathrm{CO}_{2}}} \text {, for Reaction (2) } \\
& a_{c}=k_{3} \frac{{ }_{\mathrm{CH}_{4}}}{\left(\mathrm{P}_{\mathrm{H}_{2}}\right)^{2}} \text {, for Reaction (3) } \\
& a_{c}=\left(k_{4}\right)^{\frac{1}{2}}\left(\frac{\mathrm{P}_{\mathrm{CH}_{4}} \cdot P_{\mathrm{CO}}}{\mathrm{P}_{\mathrm{H}_{2} \mathrm{O}} \cdot \mathrm{P}_{\mathrm{H}_{2}}}\right)^{\frac{1}{2}} \text {, for Reaction (4) }
\end{aligned}
$$

The carbon activity in the gas phase for each reaction has been calculated and plotted in terms of temperatures and gas chemistries, as illustrated in Figs. 5 through 8. Also plotted in the figures are equilibrium carbon activities in austenitic alloys, which are assumed to be in equilibrium with $\mathrm{Cr}_{23} \mathrm{C}_{6}$. This is a reasonable assumption, since $\mathrm{Cr}_{23} \mathrm{C}_{6}$ carbides are the most commonly observed carbides in austenitic alloys. The carbon activity maps thus constructed allow prediction of whether or not carburization of metals is thermodynamically feasible. When the carbon activity in the gas phase (i.e., helium environment) is greater than the carbon activity in the metal, carburization of the metal is thermodynamically feasible; conversely, decarburization of the metal is expected. The experimental results were then compared with the thermodynamic predictions made by these carbon activity maps. This comparison can reveal information regarding the possible dominating chemical reaction (or reactions) that controls the gas-metal reaction with regard to carburization or decarburization. This method of analysis was discussed in detail in a previous paper (Ref. 1).

Carburization was observed in all four environments at the temperatures tested [i.e., $649^{\circ}$ to $1000^{\circ} \mathrm{C}\left(1200^{\circ}\right.$ to $\left.1832^{\circ} \mathrm{F}\right)$ ]. The rates of carburization were observed to increase with increasing temperatures. These experimental observations were then compared with the thermodynamic 


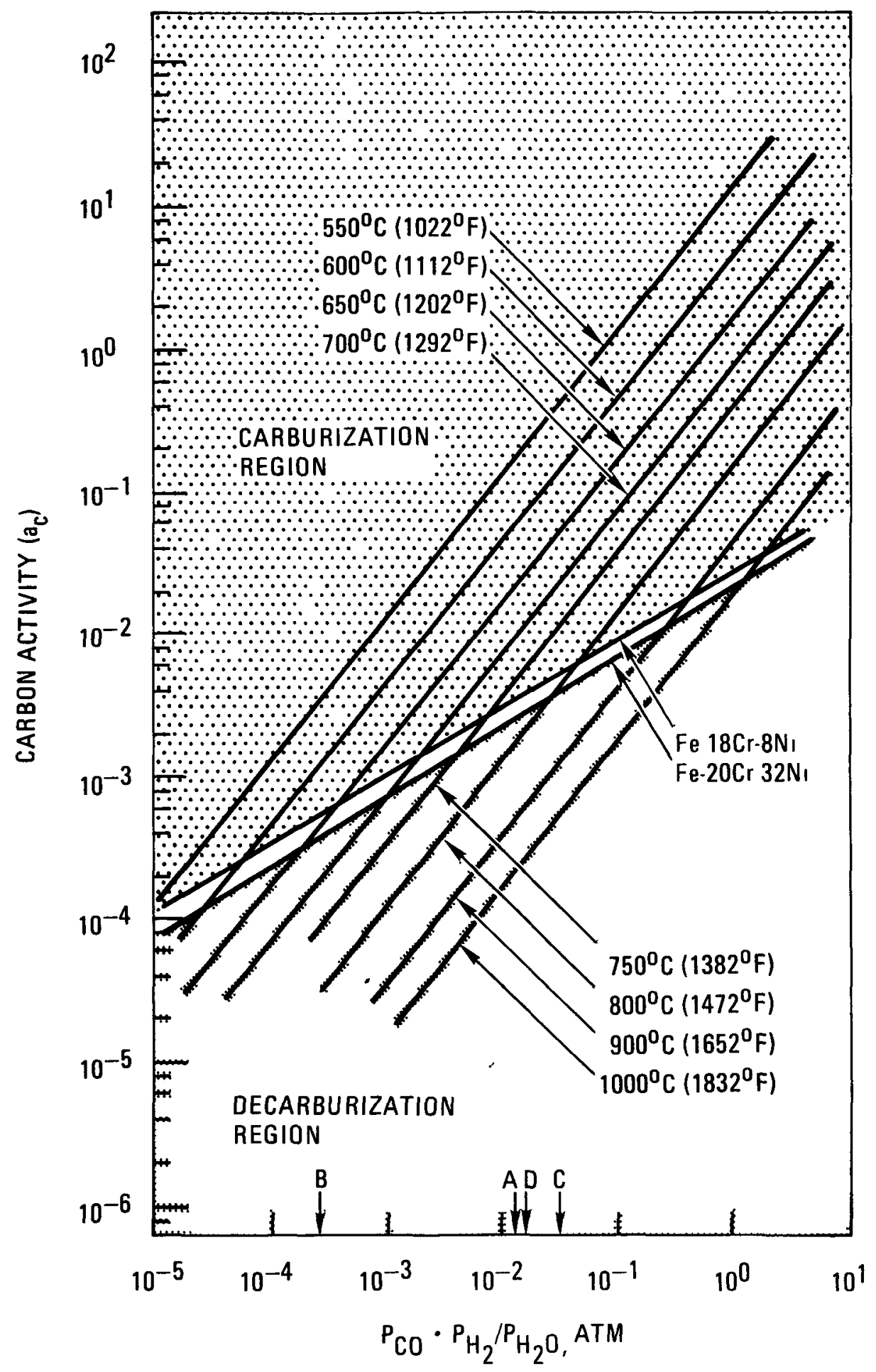

Fig. 5. Calculated carbon activities in the test environment for various temperatures based on the reaction: $\mathrm{CO}+\mathrm{H}_{2} \rightleftharpoons \mathrm{C}+\mathrm{H}_{2} \mathrm{O}$. Also plotted are calculated carbon activities in equilibrium with $\mathrm{Cr}_{23} \mathrm{C}_{6}$ in indicated alloys. 


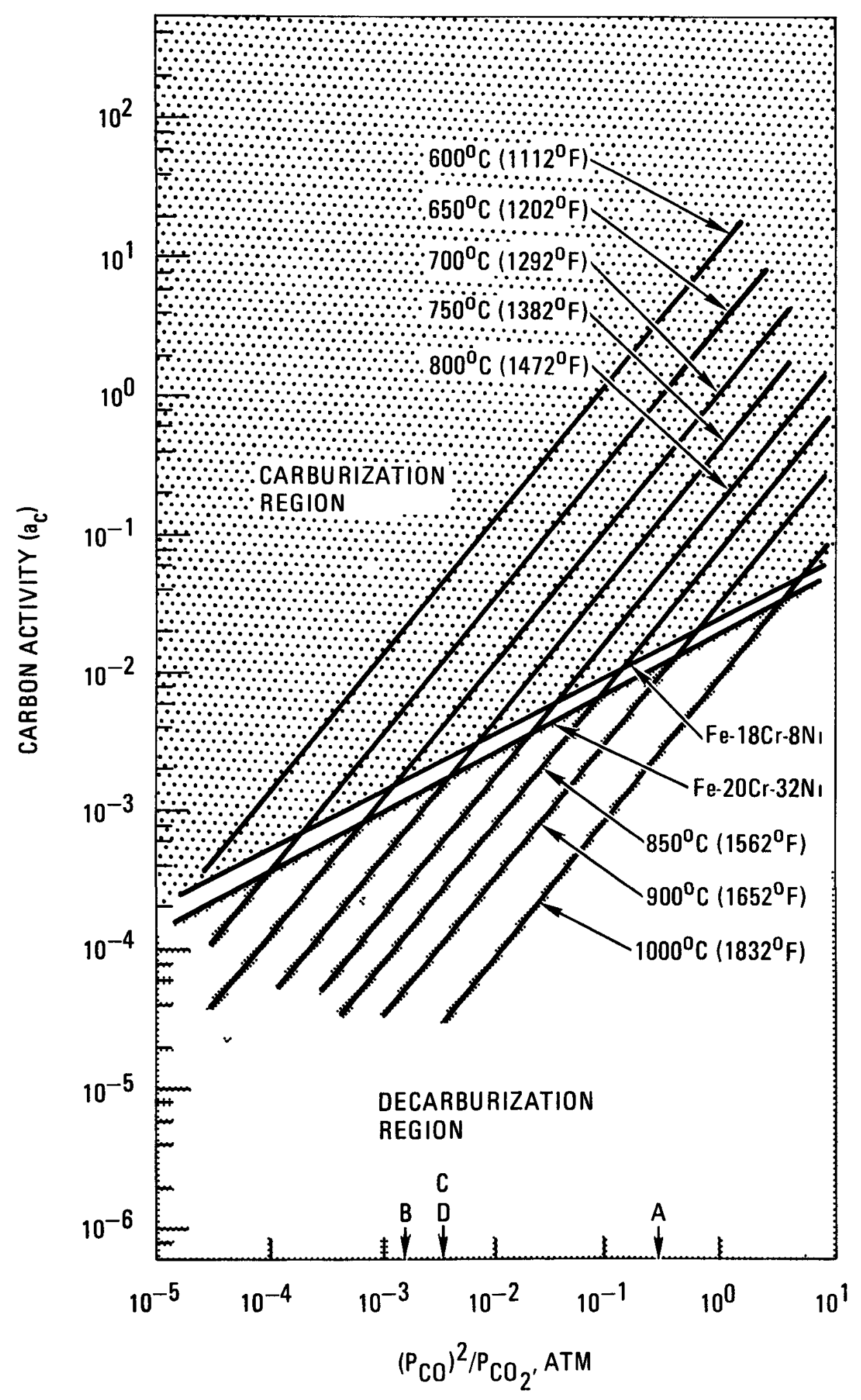

Fig. 6. Calculated carbon activities in the test environment for various temperatures based on the reaction: $2 \mathrm{CO} \rightleftharpoons \mathrm{C}+\mathrm{CO}_{2}$. Also plotted are calculated carbon activities in equilibrium with $\mathrm{Cr}_{23} \mathrm{C}_{6}$ in indicated alloys. 


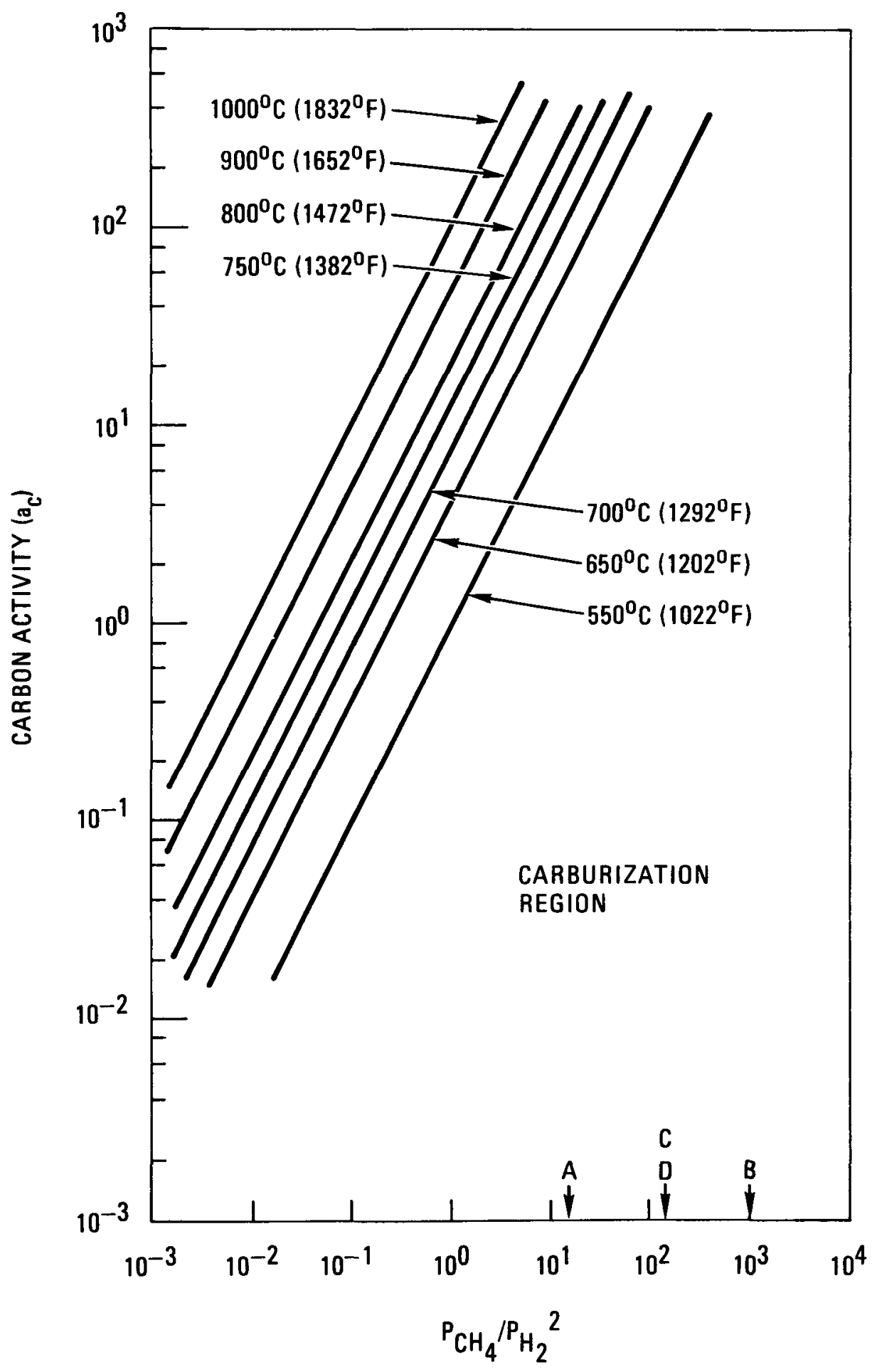

Fig. 7. Calculated carbon activities in the test environment for various temperatures based on the reaction: $\mathrm{CH}_{4} \rightleftharpoons \mathrm{C}+2 \mathrm{H}_{2}$. Carbon activities in austenitic alloys not plotted in the figure are well below the range in the figure. 


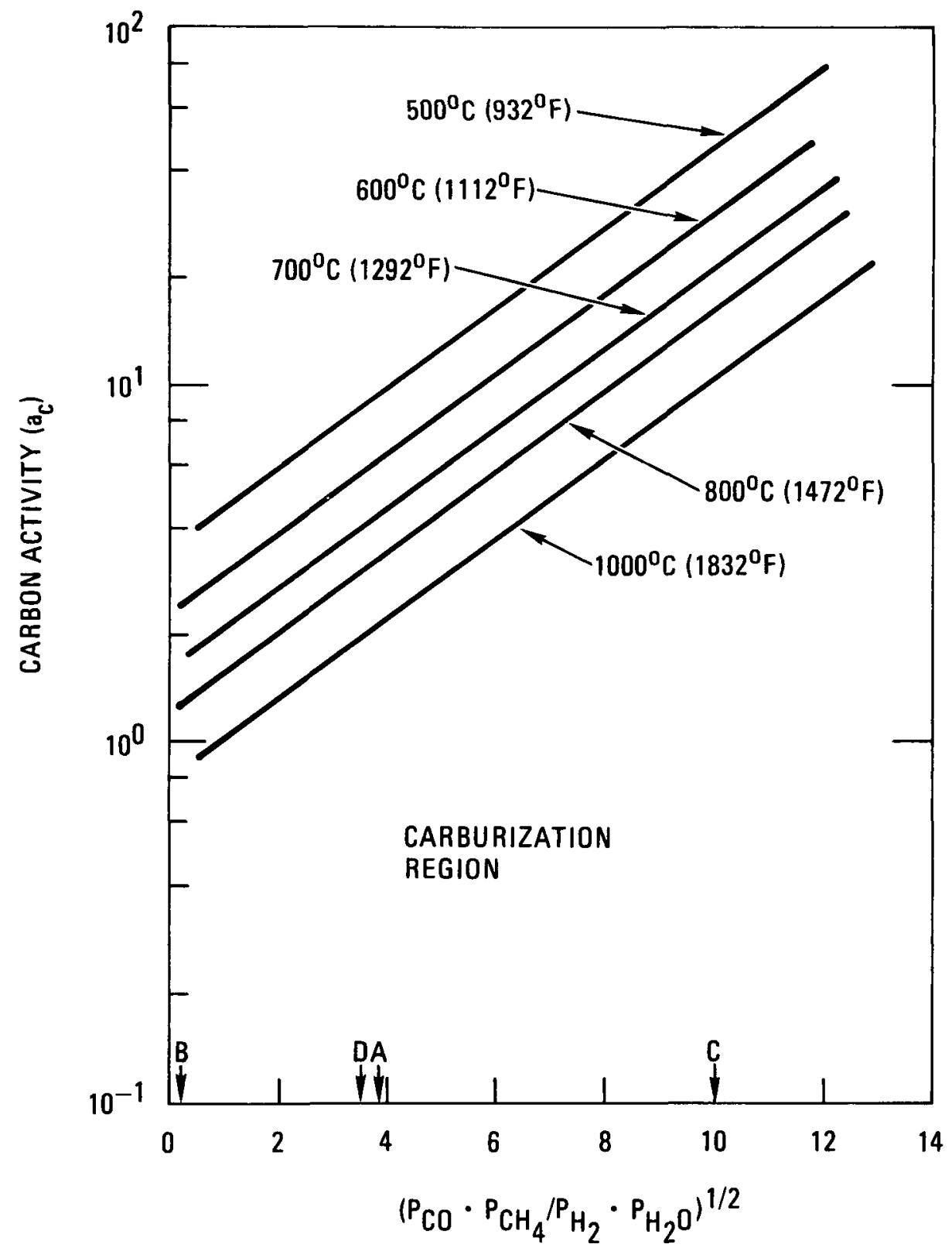

Fig. 8. Calculated carbon activities in the test environment for various temperatures based on the reaction: $\mathrm{CH}_{4}+\mathrm{CO} \rightleftharpoons 2 \mathrm{C}+\mathrm{H}_{2} \mathrm{O}+\mathrm{H}_{2}$. Carbon activities in austenitic alloys not plotted in the figure are well below the range in the figure. 
predictions using the carbon activity maps. Reaction (1) ( $\mathrm{CO}+\mathrm{H}_{2} \rightleftharpoons \mathrm{C}+$ $\mathrm{H}_{2} \mathrm{O}$ ), as illustrated in Fig. 5, predicts decarburization at all test temperatures in Environment $B$ and at temperatures above approximately $750^{\circ}$ to $800^{\circ} \mathrm{C}\left(1382^{\circ}\right.$ to $\left.1472^{\circ} \mathrm{F}\right)$ for Environments $\mathrm{A}, \mathrm{C}$ and $\mathrm{D}$. This is not consistent with the experimental observations, and reaction (1) is thus believed to play no part in the carburization of metals in the temperature range of interest. Reaction (2) $\left(2 \mathrm{CO} \rightleftharpoons \mathrm{CO}_{2}+\mathrm{C}\right)$ predicts carburization at temperatures up to approximately $850^{\circ} \mathrm{C}\left(1562^{\circ} \mathrm{F}\right)$ and decarburization at $900^{\circ} \mathrm{C}\left(1650^{\circ} \mathrm{F}\right)$ and above for Environment A. Decarburization is predicted for Environment $B$ at temperatures above approximately $706^{\circ} \mathrm{C}\left(1300^{\circ} \mathrm{F}\right)$, and for Environments $\mathrm{C}$ and $\mathrm{D}$ at temperatures above approximately $760^{\circ} \mathrm{C}\left(1400^{\circ} \mathrm{F}\right)$. The predictions are, again, not in total agreement with the experimental observations. Thus, reaction (2) is not considered to be a dominant reaction controlling the carburizationdecarburization reactions for the alloys tested. Carbon activities $\left(a_{c}\right)$ for reactions (3) and (4) in all four test environments at all test temperatures were orders of magnitude higher than those of the alloys, as illustrated in Figs. 7 and 8. Carburization is predicted at all test temperatures by reactions (3) and (4). This is consistent with the experimental observations. Therefore, reactions (3) and/or (4) are believed to control the carburization reactions.

Carburization of austenitic alloys is also thermodynamically possible through reaction (5) below:

$$
6 \mathrm{CO}+27 \mathrm{Cr} \rightleftharpoons \mathrm{Cr}_{23} \mathrm{C}_{6}+2 \mathrm{Cr}_{2} \mathrm{O}_{3}
$$

This reaction results in simultaneous carburization and oxidation of chromium in the alloy by $\mathrm{CO}$. Equilibrium CO partial pressures $\left(\mathrm{P}_{\mathrm{CO}}\right)$ for the reaction for chromium activities $\left(\mathrm{a}_{\mathrm{Cr}}\right)$ of 0.5 and 1.0 are shown in Fig. 9. [Chromium activites of 0.5 to 1.0 represent an approximate range for Alloy $800 \mathrm{H}$ and Hastelloy Alloy $\mathrm{X}$ in a temperature range of interest (Ref. 8); somewhat lower values of ${ }^{a} \mathrm{Cr}$ for Inconel Alloy 617 are expected.] Figure 9 predicts carburization in Environments $A$ and $B$ at all test temperatures $\left[900^{\circ} \mathrm{C}\left(1650^{\circ} \mathrm{F}\right)\right.$ and lower] and in Environments $\mathrm{C}$ and $\mathrm{D}$ 


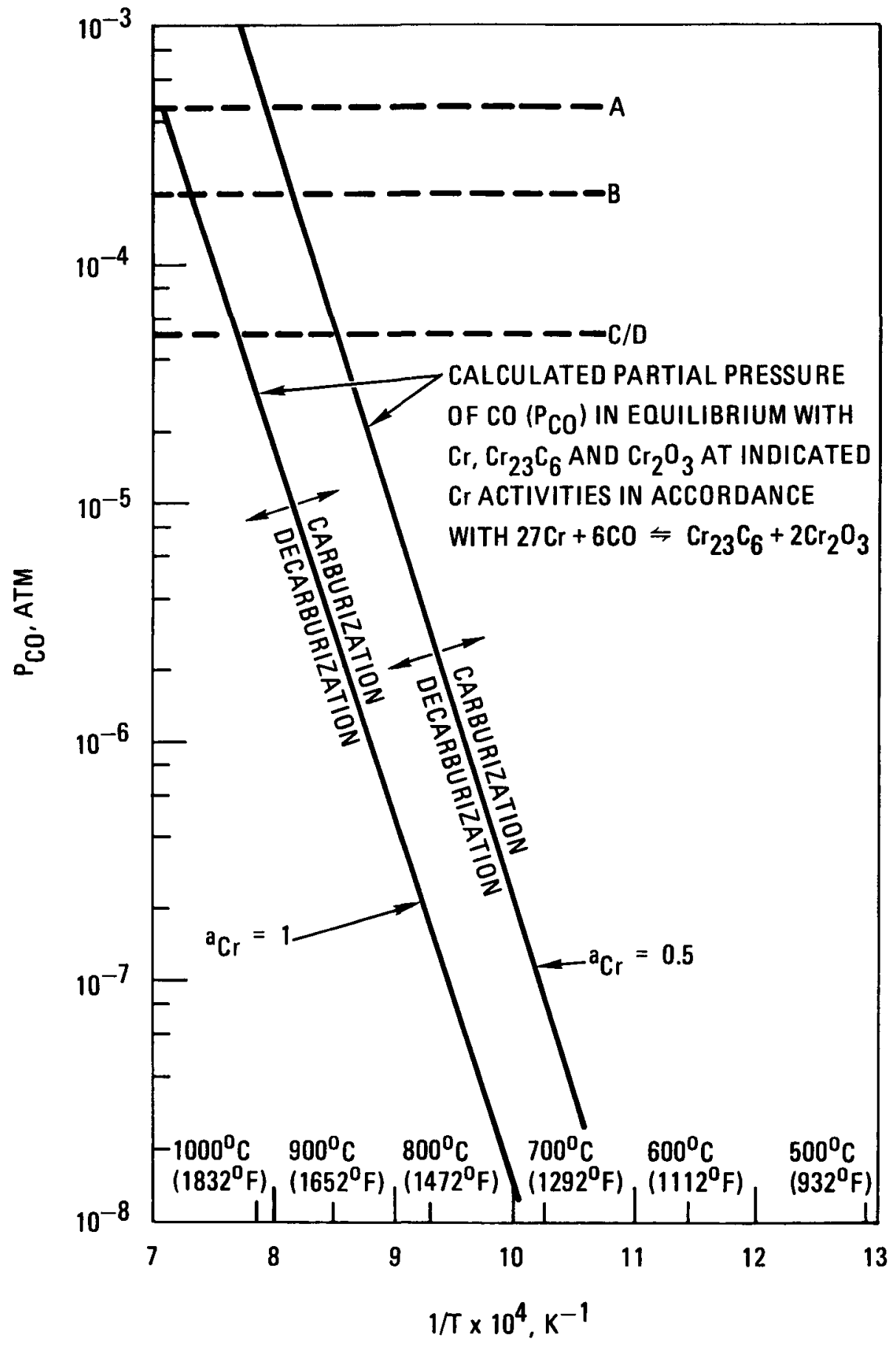

Fig. 9. Calculated partial pressure of $\mathrm{Co}$ in equilibrium with $\mathrm{Cr}, \mathrm{Cr}_{23} \mathrm{C}_{6}$ and $\mathrm{Cr}_{2} \mathrm{O}_{3}$ at indicated $\mathrm{Cr}$ activities in accordance with the reaction $27 \mathrm{Cr}+6 \mathrm{CO}=\mathrm{ir}_{23} \mathrm{C}_{6}+2 \mathrm{Cr}_{2} \mathrm{O}_{3}$ as a function of $1 / \mathrm{T}$. Also plotted are partial pressures of $\mathrm{CO}$ in Environments A, B, $\mathrm{C}$ and $\mathrm{D}$. 
at test temperatures below $900^{\circ} \mathrm{C}\left(1650^{\circ} \mathrm{F}\right)$. At test temperatures of $900^{\circ}$ and $1000^{\circ} \mathrm{C}\left(1650^{\circ}\right.$ and $\left.1832^{\circ} \mathrm{F}\right)$, Environments $\mathrm{C}$ and $\mathrm{D}$ could either carburize or decarburize the alloy depending on its chromium activity $\left(\mathrm{a}_{\mathrm{Cr}}\right)$. It is thus tentatively concluded that Reaction (5) may also be important in the carburization process.

In addition to carburization-decarburization reactions, oxidation of metals by gaseous impurities in helium may also occur. Either one or both of the following two chemical reactions can be responsible for the oxidation:

$$
\begin{aligned}
& \mathrm{H}_{2} \mathrm{O} \rightleftharpoons 1 / 20_{2}+\mathrm{H}_{2} \\
& \mathrm{CO}_{2} \rightleftharpoons 1 / 20_{2}+\mathrm{Co}
\end{aligned}
$$

The oxygen potential $\left(\mathrm{P}_{\mathrm{O}_{2}}\right)$ of the test environment can be determined from the above two reactions through their equilibrium constants (k) as shown below:

$$
\begin{aligned}
& \mathrm{k}_{6}=\frac{\left(\mathrm{P}_{\mathrm{O}_{2}}\right)^{\frac{1}{2}} \cdot \mathrm{P}_{\mathrm{H}_{2}}}{\mathrm{P}_{\mathrm{H}_{2} \mathrm{O}}} \text {, for reaction (6) } \\
& \mathrm{k}_{7}=\frac{\left(\mathrm{P}_{\mathrm{O}_{2}}\right)^{\frac{1}{2}} \cdot \mathrm{P}_{\mathrm{CO}}}{\mathrm{P}_{\mathrm{CO}_{2}}} \text {, for reaction (7) }
\end{aligned}
$$

No $\mathrm{CO}_{2}$ was deliberately added to Environment $\mathrm{A}$ and only a little

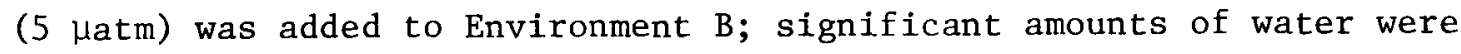
present in both $\mathrm{A}$ and $\mathrm{B}$ environments. The calculated oxygen potential based on Reaction (6) for both $A$ and $B$ environments was significantly higher than that based on Reaction (7). Thus, the oxygen potential in both $A$ and $B$ environments is believed to be dominated by Reaction (6) or the $\mathrm{P}_{\mathrm{H}_{2} \mathrm{O}} / \mathrm{P}_{\mathrm{H}_{2}}$ ratio. For Environments $\mathrm{C}$ and $\mathrm{D}, \mathrm{H}_{2} \mathrm{O}$ and $\mathrm{CO}_{2}$ were not 
deliberately added to the test gas. The concentration of $\mathrm{CO}_{2}$ in both $\mathrm{C}$ and $\mathbf{D}$ environments was not measurable by a gas chromatograph. For calculation of the oxygen potential in Reaction (7), $0.5 \mu \mathrm{atm}$ of $\mathrm{CO}_{2}$ (detection limit of the gas chromatograph) was assumed. $\mathrm{H}_{2} \mathrm{O}$ was not measurable in Environment $C$, and 0.5 Hatm was assumed in the calculation of oxygen potential in Reaction (6). Environment D contained a very low concentration $(1.5 \mu \mathrm{atm})$ of $\mathrm{H}_{2} 0$. It is shown from the calculation of the oxygen potential from Reactions (6) and (7) that either reaction could be dominating the oxygen potential for $C$ and $D$ environments. It is assumed in this paper that Reaction (6) or $\mathrm{P}_{\mathrm{H}_{2}} \mathrm{O} / \mathrm{P}_{\mathrm{H}_{2}}$ ratios controlled the oxygen potential of $\mathrm{C}$ and $\mathrm{D}$ environments.

The oxides that are thermodynamically capable of forming on these alloys are shown in Fig. 10, where the oxygen partial pressure $\left(\mathrm{P}_{0_{2}}\right)$ in equilibrium with the oxide is plotted as a function of temperature. Also included in the figure are calculated oxygen potentials of test environments based on Reaction (6) or $\mathrm{P}_{\mathrm{H}_{2} \mathrm{O}} / \mathrm{P}_{\mathrm{H}_{2}}$ ratios. Oxidation of those elements in the alloy is thermodynamically feasible when $\mathrm{P}_{0_{2}}$ in the test environment is greater than those in equilibrium with the oxides. All four environments are reducing to iron oxdies (and less stable oxides of nickel, molybdenum, cobalt, etc.) and oxidizing to chromium and elements more reactive than chromium (silicon, titanium, aluminum, etc.). $\mathrm{Cr}_{2} \mathrm{O}_{3}$ oxides are at the borderline at high temperatures in $C$ and $D$ environments, which exhibit a much lower oxygen potential.

It is clear from the foregoing discussion that the gas-metal reactions are controlled by both oxygen and carbon potentials in the test environment. Depending on their combined potentials, either oxidation or carburization, or both, can occur at the gas-metal interface to form a scale. The type of reactions and corrosion products can be approximately predicted by thermodynamic stability diagrams. The most important system in oxidation/ carburization of austenitic alloys in these high-temperature gaseous 


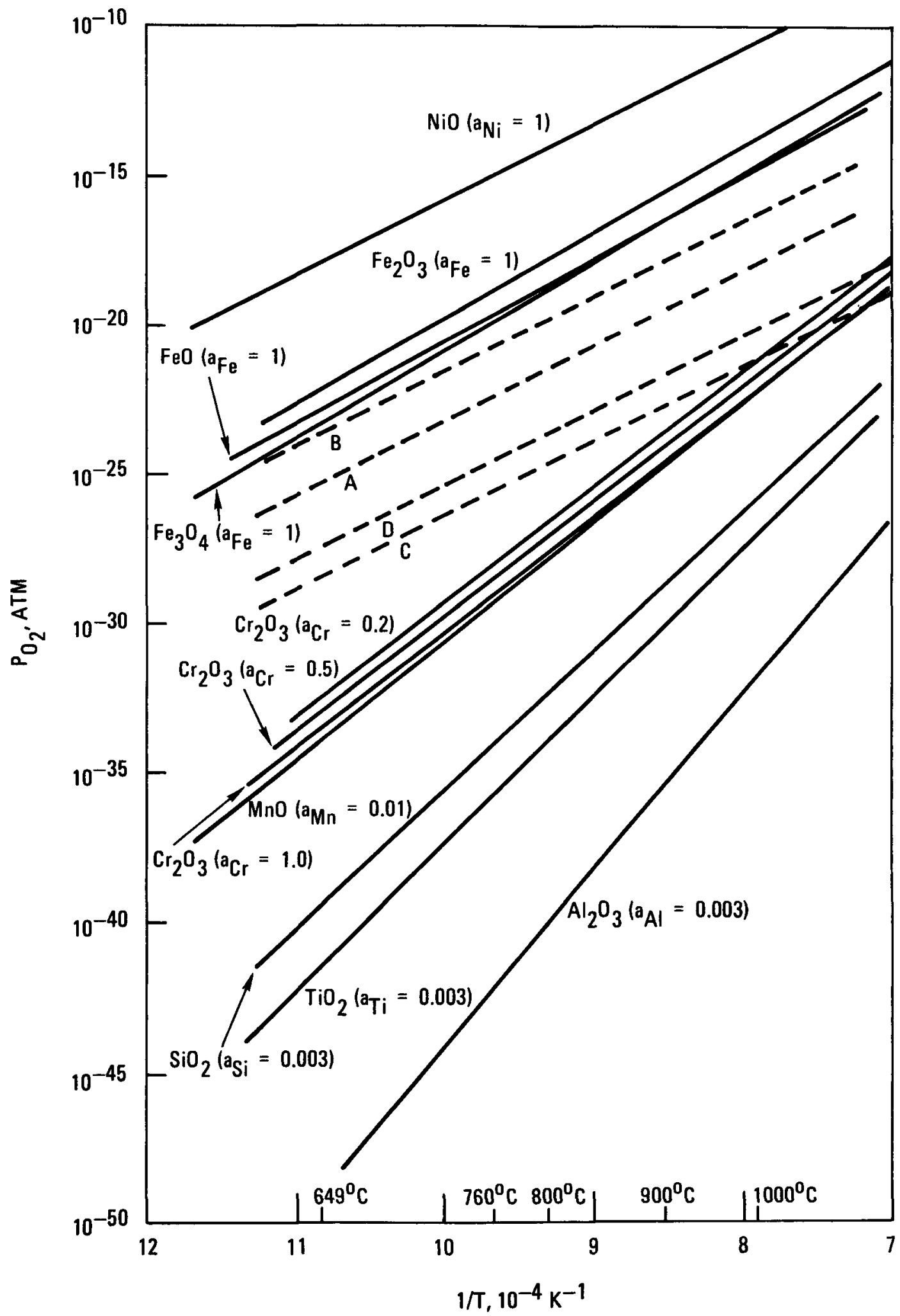

Fig. 10. Equilibrium oxygen partial pressures for various metallic oxides as a function of $1 / T\left(K^{-1}\right)$. Also plotted in the figure are calculated oxygen partial pressures for Environments A, B, C and $D$ based on the reaction: $\mathrm{H}_{2} \mathrm{O} \rightleftharpoons 1 / 2 \quad \mathrm{O}_{2}+\mathrm{H}_{2}$. 
environments is the $\mathrm{Cr}-\mathrm{O}-\mathrm{C}$ system. The stability diagram of the $\mathrm{Cr}-\mathrm{O}-\mathrm{C}$ system at $900^{\circ} \mathrm{C}\left(1650^{\circ} \mathrm{F}\right)$ is illustrated in Fig. 11. The stability diagram defines the region in which the phases are stable and are expected to form at the gas-metal interface.

The present investigation showed carburization of alloys in all four environments. Carbon activities in these environments are thus believed to be equal to or greater than those in equilibrium with $\mathrm{Cr}_{23} \mathrm{C}_{6}$ carbides, otherwise carburization should not have occurred (Fig. 11). Therefore, in the present discussion of oxidation and carburization of metals, it is sufficient to consider only the oxide-carbide boundary in the thermodynamic stability diagrams such as the one shown in $\mathrm{Fig} .11$. At $900^{\circ} \mathrm{C}\left(1650^{\circ} \mathrm{F}\right)$, for example, the oxygen potentials in Environments $A$ and $B$ are orders of magnitude higher than oxygen partial pressures in equilibrium with both $\mathrm{Cr}_{2} \mathrm{O}_{3}$ oxides and chromium carbides (i.e., $\mathrm{Cr}_{23} \mathrm{C}_{6}, \mathrm{Cr}_{7} \mathrm{C}_{3}$ and $\mathrm{Cr}_{3} \mathrm{C}_{2}$ ). $\mathrm{Cr}_{2} \mathrm{O}_{3}$ oxides are thus predicted to form on the alloy surface at the gas-metal interface. This is confirmed in the present investigation.

Figure 12 shows typical oxide scales formed on the alloy surface of Alloy 800H, Inconel Alloy 617 and Hastelloy Alloy $X$ after exposure to Environments $A$ and $B$. The scale analyzed by $X$-ray diffraction was found to consist primarily of $\mathrm{Cr}_{2}{ }^{0}{ }_{3}$ and $\mathrm{MnCr}_{2}{ }^{0}{ }_{4}$ spinels for all three alloys. Carburization of the metal underneath the scale can only occur when carbon penetrates through the oxide scale.

For Environments $C$ and $D$, the oxygen potentials either are slightly above or intersect the oxide-carbide boundary. Either oxides or carbides are thus expected to form on the alloy surface. It was observed experimentally that after exposure to these environments ( $C$ and $D$ ), the scale formed on the alloy surface of these three alloys consisted of oxides after short-time exposure $(\geq 1000 \mathrm{~h})$ and of oxides and carbides after longterm exposure $(\geq 3000 \mathrm{~h})$ at $800^{\circ}, 900^{\circ}$ and $1000^{\circ} \mathrm{C}\left(1472^{\circ}, 1650^{\circ}\right.$, and $\left.1832^{\circ} \mathrm{F}\right)$. Figure 13 shows typical scales consisting of oxides and 


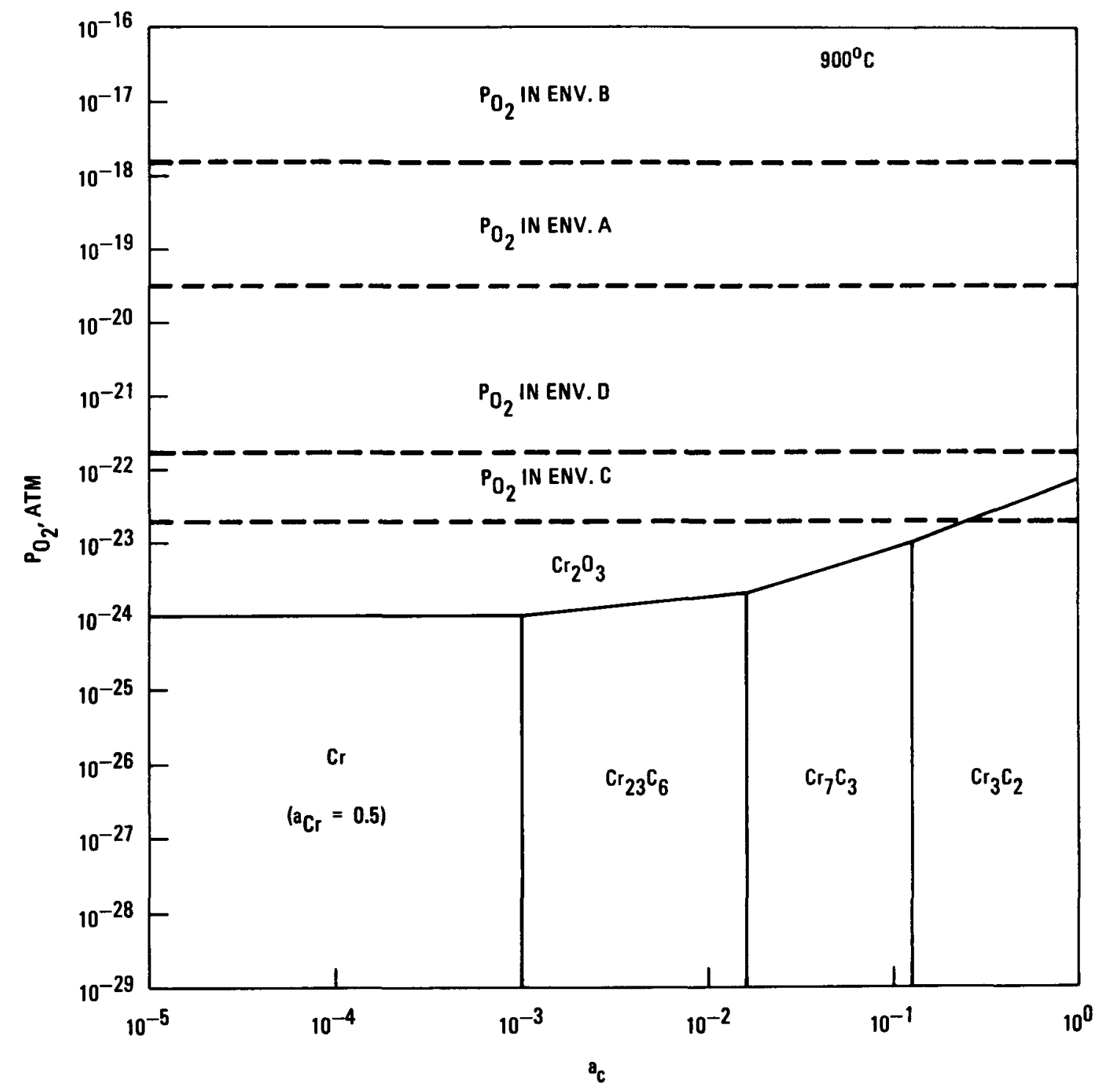

Fig. 11. The stability diagram for the $\mathrm{Cr}-0-\mathrm{C}$ system at $900^{\circ} \mathrm{C}\left(1650^{\circ} \mathrm{F}\right)$. Also shown in the figure are calculated oxygen partial pressures in Environments A, B, C and D. 


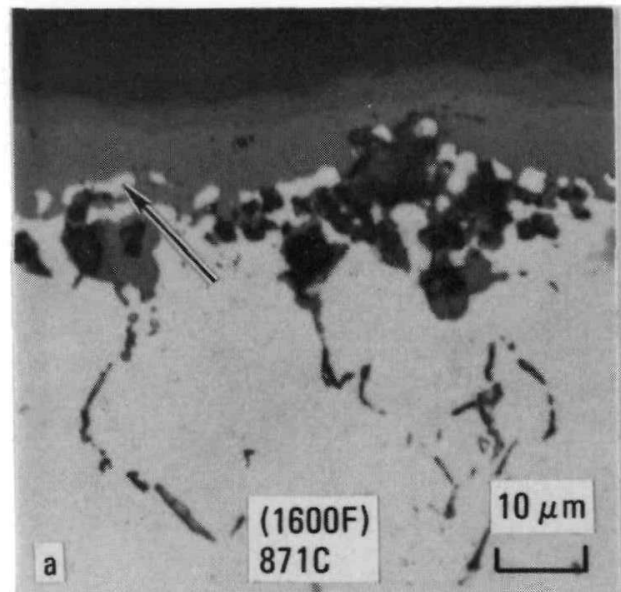

ALLOY $800 \mathrm{H}$, ENV. A

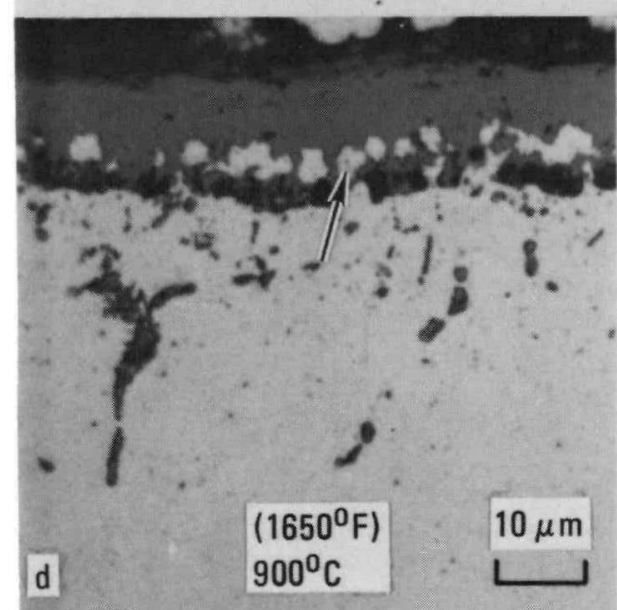

ALLOY $800 \mathrm{H}$, ENV. B

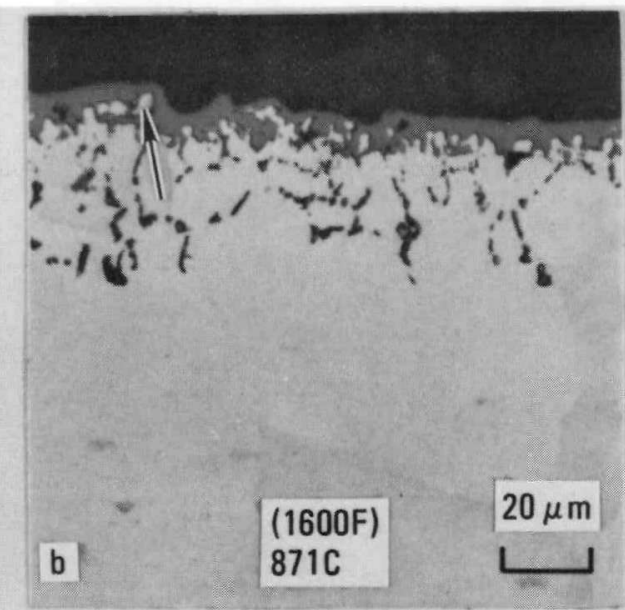

INCONEL ALLOY 617, ENV. A

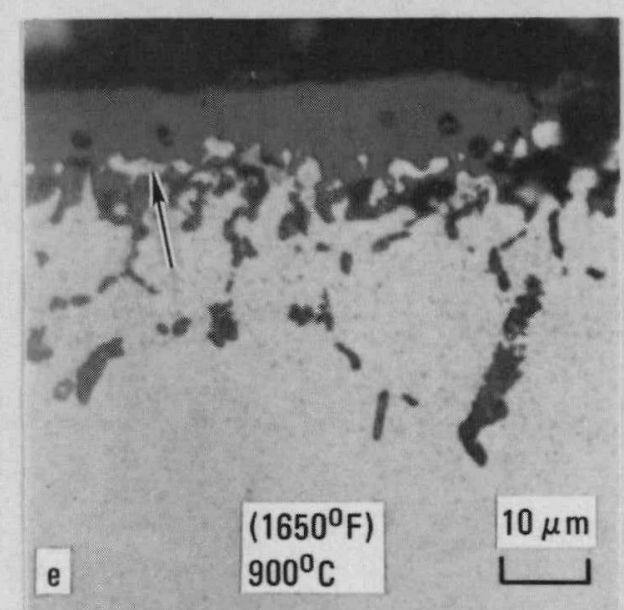

INCONEL ALLOY 617, ENV. B

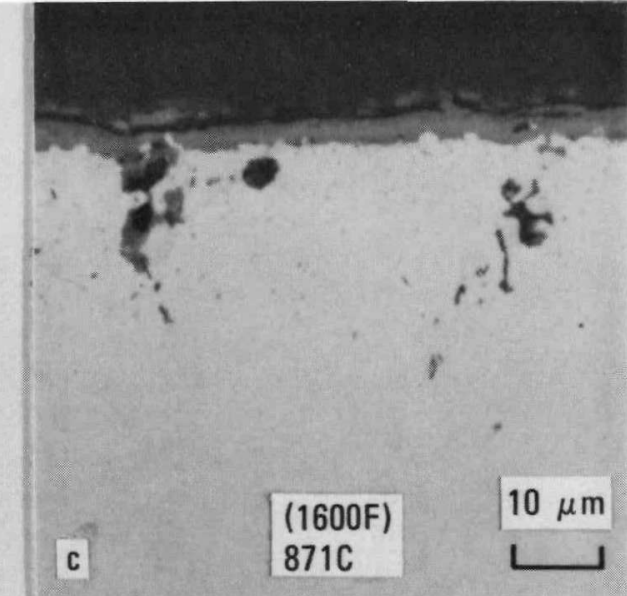

HASTELLOY ALLOY X, ENV. A

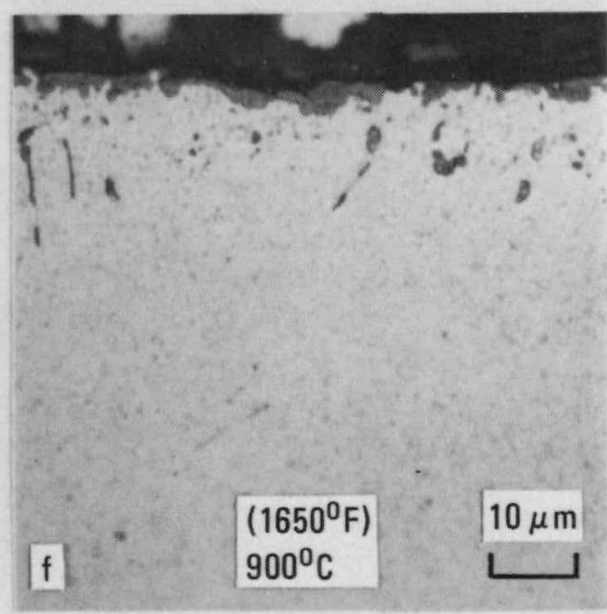

HASTELLOY ALLOY X, ENV. B

Fig. 12. Optical photomicrographs showing the typical oxide scale formed on the metal surface of Alloy $800 \mathrm{H}$, Inconel Alloy 617 and Hastelloy Alloy X after exposure to Environment A at $871^{\circ} \mathrm{C}\left(1600^{\circ} \mathrm{F}\right)$ for $5000 \mathrm{~h}(\mathrm{a}, \mathrm{b}, \mathrm{c})$ and to Environment $\mathrm{B}$ at $900^{\circ} \mathrm{C}\left(1650^{\circ} \mathrm{F}\right)$ for $5000 \mathrm{~h}$ $(d, e, f)$. The phases indicated by the arrow were found to be chromium-depleted metal matrix phases. Unetched. 


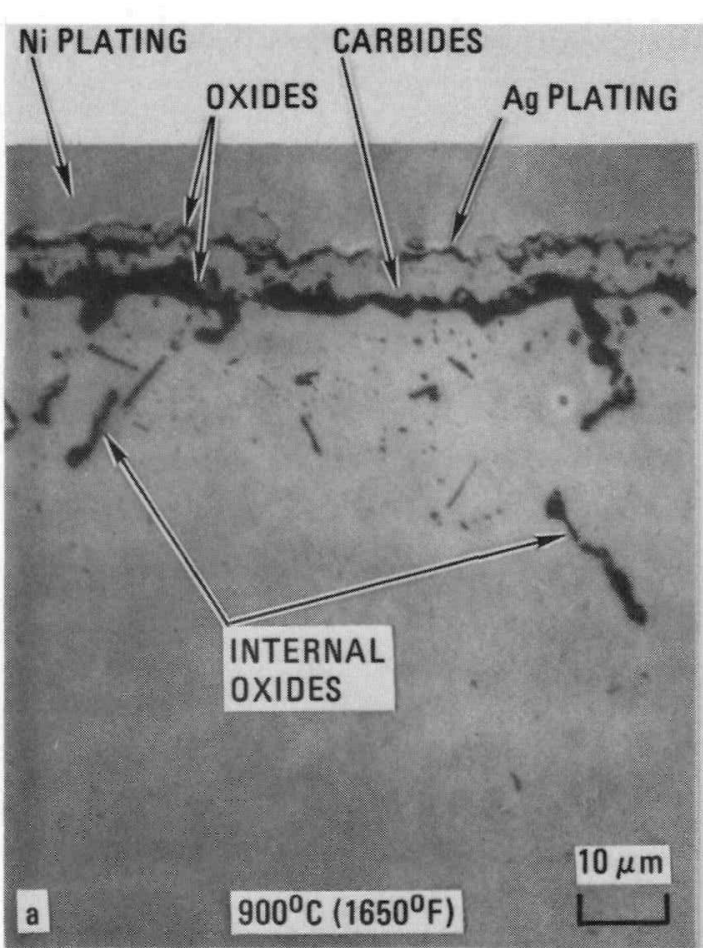

ALLOY $800 \mathrm{H}$, ENV. C

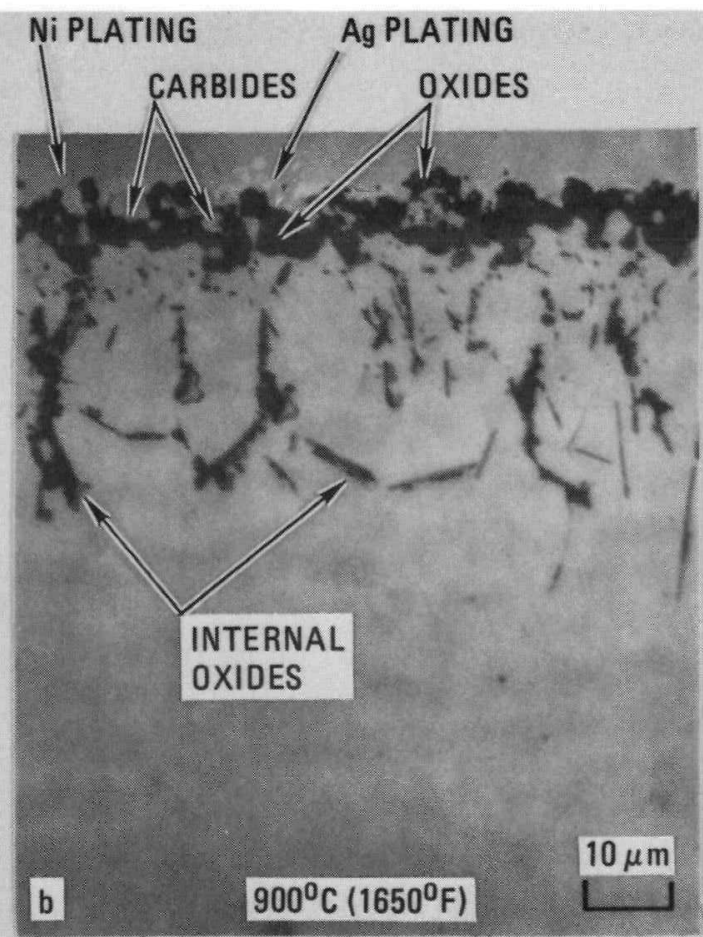

INCONEL ALLOY 617, ENV. C

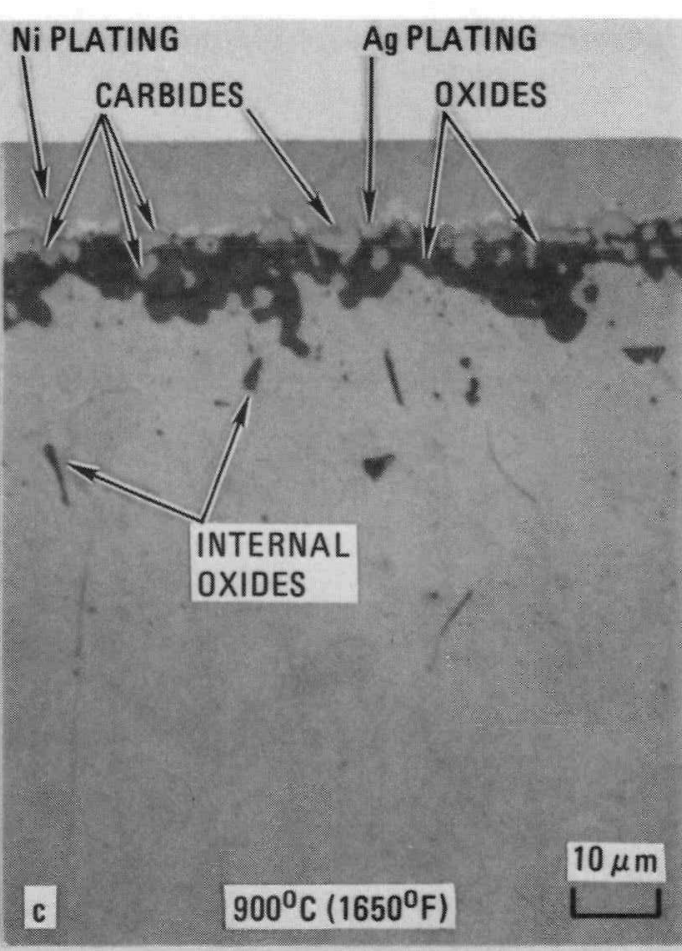

HASTELLOY ALLOY X, ENC. C

Fig. 13. Optical photomicrographs showing the typical oxide-carbide duplex scale formed on the metal surface of Alloy $800 \mathrm{H}$ (a). Inconel Alloy 617 (b) and Hastelloy Alloy X (c) after exposure to Environment $\mathrm{C}$ at $900^{\circ} \mathrm{C}\left(1650^{\circ} \mathrm{F}\right)$ for $3000 \mathrm{~h}$. Unetched. 
carbides formed on specimen surfaces. The oxides in the scale were found by electron microprobe analysis to be enriched in $\mathrm{Cr}$ and $\mathrm{Mn}$. X-ray diffraction analysis indicated that the oxides in the scale consisted primarily of $\mathrm{Cr}_{2} \mathrm{O}_{3}$ and $\mathrm{MnCr}_{2} \mathrm{O}_{4}$. The following hypothesis can be offered to explain the presence of oxides and carbides in the scale when the oxygen potential in the environment is at or near the borderline. Assuming $\mathrm{P}_{2}$ in the environment is slightly above the borderline, oxides are expected to form initially on the metal surface. After nucleation and some growth of the oxides, $\mathrm{P}_{0_{2}}$ at the nearby areas may decrease to favor the formation of carbides until the formation of oxides is again favored. The experimental results of the present investigation appear to suggest this type of repetitive growth process for the scale formation in Environments C and D (Fig. 13).

Previously, it was concluded that Reaction (3) and/or (4) may be controlling the carburization reactions, and that Reaction (5) may also be important in these reactions. Considering Reaction (3), the carbon activities of the four environments at the test temperatures are many orders of magnitude higher than those in the alloys. The carbon activities in Environment $B$ (one of the high-oxygen potential environments) are higher than those of $C$ and $D$ environments (low-oxygen potential environments) at al1 test temperatures. For Reaction (4) the carbon activites, although slightly higher in Environment $C$, are about the same in $A$ and $D$ environments. As for Reaction (5), carburization potential in A or $B$ environment is higher than that of $C$ and $D$ environments. Therefore, it is believed that the differences in carburization rates between A/B (highoxygen potential) and C/D (low-oxygen potential) environments are not due to differences in the thermodynamic carburization potential but are related to kinetic effects.

As discussed previously, the scale formed in $A$ and $B$ environments (high-oxygen potential) consisted mainly of $\mathrm{Cr}_{2} \mathrm{O}_{3}$ and $\mathrm{MnCr}_{2} \mathrm{O}_{4}$ oxides. The oxide scale was found by optical microscopy and scanning electron 
microscopy to be dense and adherent. It is believed that carburization of the metal underneath the oxide scale can occur only when carbon penetrates through the scale.

A scale consisting of oxides and carbides was observed to form on the alloy surfaces in $C$ and $D$ environments (low-oxygen potential). The oxides in the scale were found to be $\mathrm{Cr}_{2} \mathrm{O}_{3}$ and $\mathrm{MnCr}_{2} \mathrm{O}_{4}$. Similar to the specimens exposed in $A$ and $B$ environments, carbon coming from the test environment would have to penetrate through the scale to carburize the metal underneath. Nevertheless, carbides in the scale may become additional sources of carbon to carburize the metal underneath the scale. The oxygen potential of $C$ and $D$ environments was near the borderline of the oxide-carbide boundary. Thus, carbides formed in the scale are in a metastable condition and can convert to oxides whenever the local oxygen potential increases above the $\mathrm{P}_{\mathrm{O}_{2}}$ in equilibrium with both $\mathrm{Cr}_{2} \mathrm{O}_{3}$ oxides and carbides. It was hypothesized previously that the local oxygen potential at the gas-metal interface may fluctuate during scale formation. Whenever dissociation of carbides in the scale occurs, additional carbon atoms become available to diffuse into the metal underneath the scale resulting in more carburization. This qualitatively mechanistic model is offered to explain the enhanced carburization kinetics when the scale formed on the metal surface consists of oxides and carbides. The model is illustrated graphically in Fig. 14. The present model suggests that increasing the oxygen potential of $C$ and $D$ environments to the levels where only oxides $\left(\mathrm{Cr}_{2} \mathrm{O}_{3} / \mathrm{MnCr}_{2} \mathrm{O}_{4}\right)$ form the scale (without carbides) can significantly reduce carburization kinetics. Based on the results of the present investigation, it is suggested that controlling the oxygen potential of the service environment can be an effective means of reducing carburization of metals. 


\section{ENVIRONMENTS A/B}

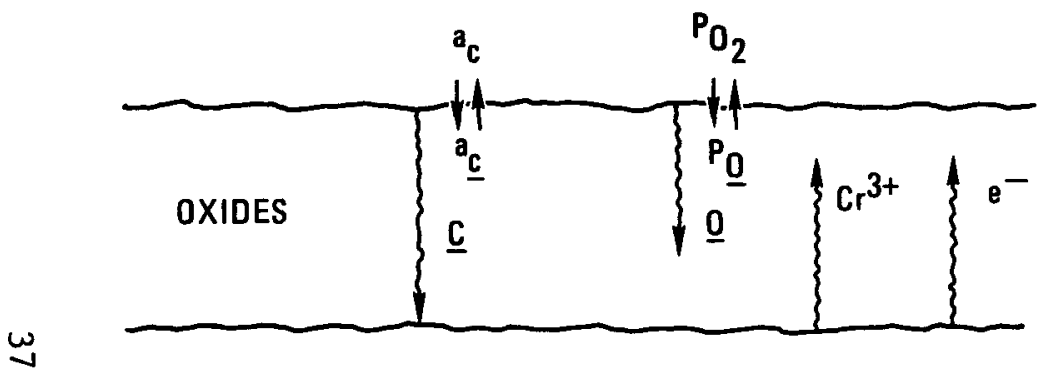

ALLOY SUBSTRATE
ENVIRONMENTS C/D

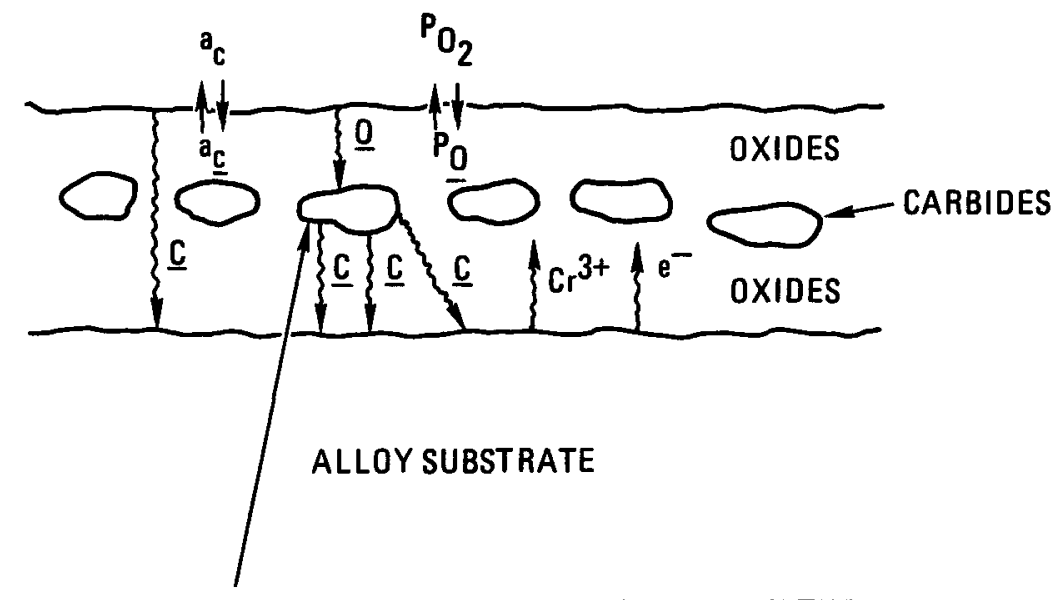

WHEN THE LOCAL OXYGEN POTENTIAL IS HIGHER THAN THE $\mathrm{P}_{\mathrm{O}_{2}}$ IN EQUILIBRIUM WITH $\mathrm{Cr}_{2} \mathrm{O}_{3}$ AND CARBIDES, THE CARBIDE DISSOCIATES RESULTING IN ADOITIONAL CARBON ATOMS PENETRATING THROUGH THE OXIDE SCALE TO CARBURIZE THE SUBSTRATE ALLOY; FOR EXAMPLE,

$2 \mathrm{Cr}_{23} \mathrm{C}_{6}+69 \underline{\underline{0}} \rightarrow 23 \mathrm{Cr}_{2} \mathrm{O}_{3}+12 \underline{\mathrm{C}}$

Fig. 14. Schematics of the qualitatively mechanistic model for carburization of metals in high-oxygen potential environments (A and $B$ ) and low-oxygen potential environments (C and D). 


\section{SUMMARY AND CONCLUSIONS}

The carburization behavior of Alloy 800H, Inconel Alloy 617 and Hastelloy Alloy $X$ in four different helium environments ( $A, B, C$ and $D$ ) at temperatures from $649^{\circ}$ to $1000^{\circ} \mathrm{C}\left(1200^{\circ}\right.$ to $\left.1832^{\circ} \mathrm{F}\right)$ for exposure times up to $10,000 \mathrm{~h}$ was studied. Environments $\mathrm{A}$ and $\mathrm{B}$ are characteristic of high-oxygen potential, while $C$ and $D$ are characteristic of low-oxygen potential. The major results and conclusions are as follows:

1. Carburization was observed in all four environments. Carburization kinetics in the low-oxygen potential environments (C and D) were found to be significantly higher, approximately an order of magnitude higher at high temperatures, than those in the highoxygen potential environments (A and B) for all three alloys. Thermodynamic analyses indicated no significant differences in the thermodynamic carburization potential between low- and high-oxygen potential environments. It is thus believed that the enhanced carburization kinetics observed in the low-oxygen potential environments are related to kinetic effects.

2. The scale formed on the metal surface in the high-oxygen potential environments consisted of oxides $\left(\mathrm{Cr}_{2} \mathrm{O}_{3}\right.$ and $\left.\mathrm{MnCr}_{2}{ }_{4}\right)$, while the scale formed in the low-oxygen potential environments consisted of oxides and carbides. The carbides formed in the scale in the low-oxygen potential environments are believed to be in a metastable condition in those environments. Whenever dissociation of carbides in the scale occurs due to increases in local oxygen potential, additional carbon atoms become available to carburize the metal underneath the scale. This qualitatively mechanistic model, illustrated graphically in Fig. 14, is proposed to explain the enhanced carburization kinetics observed in the low-oxygen potential environments. 
The present model suggests that increasing the oxygen potential of $\mathrm{C}$ and $\mathrm{D}$ environments to levels where only oxides form in the scale (without carbides) can significantly reduce carburization kinetics.

3. The present results suggest that controlling the oxygen potential of the service environment can be an effective means of reducing carburization of austenitic alloys. The effect of increasing the oxygen potential on the corrosion rates of HTGR core graphite must be considered in evaluating the practical application of this approach. 


\section{ACKNOWLEDGMENTS}

The authors are grateful to R. B. Akins and R. Hansen for conducting experiments; S. Liang, J. Knipping and R. Speas for optical metallography; D. Wall for SEM analysis; J. Wattier for statistical analysis of kinetic data; S. N. Rosenwasser and D. I. Roberts for reviewing the manuscript; J. Jayne for technical editing; and W. Ccx and her colleagues who typed the manuscript.

The work was funded by the U.S. Department of Energy under Contract DE-AT03-76ET35300. 


\section{REFERENCES}

1. F. N. Mazandarany and G. Y. Lai, Nuclear Technology, 1979, vo1. 43, pp. 349-365.

2. S. N. Rosenwasser and W. R. Johnson, "Gas-Turbine HTGR Materials Screening Test Program - Interim Results," ERDA Report GA-A13931, General Atomic Company, July 1976.

3. S. N. Rosenwasser and W. R. Johnson, "Gas Turbine and Advanced - HTGR Materials Screening Test Program," DOE Report GA-A14778, General Atomic Company, January 2978.

4. M. R. Warren and H. G. A. Bates, High Temperature Materials Program Report 23, November 1978.

5. R. H. Cook and L. W. Graham, Proceedings Petten International Conference on Alloy 800, J. R. C. Petten Establishment, Petten (NH), The Netherlands, March 1978, pp. 309-329.

6. K. G. E. Brenner and L. M. Nilsen, High Temperature Materials Program, Report 29, August 1978.

7. H. Inouye, Oak Ridge National Laboratory, private communication, September 1979.

8. F. N. Mazandaramy and R. D. Pehlke, Met. Trans. 1933, vo1. 4, pp. 2067-2076. 